\title{
Clôture intégrale et opérations de tores algébriques de complexité un dans les variétés affines
}

\author{
Kevin Langlois
}

\begin{abstract}
In this paper, we are interested in multigraded affine algebras of complexity 1 . One of the main results gives a description of integrally closed homogeneous ideals in terms of polyhedral divisors introduced by AltmannHausen. Another result allows us to compute effectively the normalization of an affine variety with an algebraic torus action of complexity one. We describe as well the integral closure of homogeneous ideals and exhibit new examples of normal homogeneous ideals.
\end{abstract}

\section{Introduction}

In this paper, we are interested in multigraded affine algebras of complexity 1 over an algebraically closed field $\mathbf{k}$ of characteristic zero.

Using the convex geometry developped by Altmann-Hausen we obtain some new results on classical questions of commutative algebra. One of our main theorems gives a description of integrally closed homogeneous ideals in terms of polyhedral divisors, see Theorem 4.6. Another result allows us to compute effectively the normalization of an affine variety with an algebraic torus action of complexity one. We describe as well the integral closure of homogeneous ideals, see Theorem 2.4, Theorem 4.2 and exhibit new examples of normal homogeneous ideals, see Theorem 5.3.

The following two classical examples illustrate well the issues that arise. Consider the algebra of Laurent polynomials in $n$ variables

$$
L_{[n]}=L_{[n]}(\mathbf{k}):=\mathbf{k}\left[t_{1}, t_{1}^{-1}, t_{2}, t_{2}^{-1}, \ldots, t_{n}, t_{n}^{-1}\right] .
$$

Notice that $L_{[n]}$ is the coordinate ring of the affine variety $\left(\mathbf{k}^{\star}\right)^{n}$. Let $A$ be a subalgebra generated by a finite number of monomials, and let $E \subset \mathbb{Z}^{n}$ be the subset of exponents corresponding to the monomials of $A$. Without loss of generality, we may suppose that $E$ generates the lattice $\mathbb{Z}^{n}$. It is known [Ho] that the normalization of $A$ is the set of linear combinations of all monomials with their exponents belonging to the rational cone $\omega \subset \mathbb{Q}^{n}$ generated by $E$. We have

$$
\bar{A}=\bigoplus_{\left(m_{1}, \ldots, m_{n}\right) \in \omega \cap \mathbb{Z}^{n}} \mathbf{k} t_{1}^{m_{1}} \ldots t_{n}^{m_{n}}
$$

where $\bar{A}$ is the normalization of $A$. For instance, if $n=1$ and if $A$ is the subalgebra generated by the monomials $t_{1}^{2}$ and $t_{1}^{3}$ then the normalization of $A$ is the polynomial ring $\mathbf{k}\left[t_{1}\right]$.

A similar problem arises for monomial ideals. Assume that the algebra $A$ is normal. Let $I$ be an ideal of $A$ generated by monomials. The convex hull in $\mathbb{Q}^{n}$ of all exponents appearing in $I$ is a polyhedron $P$ 
contained in $\omega$. This polyhedron $P$ satisfies $P+\omega \subset P$. The integral closure of $I$ is equal to

$$
\bar{I}=\bigoplus_{\left(m_{1}, \ldots, m_{n}\right) \in P \cap \mathbb{Z}^{n}} \mathbf{k} t_{1}^{m_{1}} \ldots t_{n}^{m_{n}}
$$

We can determine $P$ by means of a finite system of monomials generating the ideal $I$. For instance, if $n=2, A=\mathbb{C}\left[t_{1}, t_{2}\right]$ and if $I$ is the ideal generated by the monomials $t_{1}^{3}$ and $t_{2}^{3}$ then

$$
\bar{I}=\left(t_{1}^{3}, t_{1}^{2} t_{2}, t_{1} t_{2}^{2}, t_{2}^{3}\right) .
$$

See [LeTe], [HS], [Va] for more details concerning the integral closure of ideals; we recall below the definition. Note that the study of integrally closed ideals allows us to find the normalization of a blowing-up (see $[\mathrm{KKMS}]$ for toric varieties and $[\mathrm{Br}]$ for spherical varieties). This is used as well in order to describe (T-equivariant) affine modifications (see [KZ], [Du]).

By analogy with the monomial case presented above, we address more generally the actions of algebraic tori of complexity 1. Before formulating our results, let us recall some notation.

An algebraic torus $\mathbb{T}$ of dimension $n$ is an algebraic group isomorphic to $\left(\mathbf{k}^{\star}\right)^{n}$. Let $M$ be the character lattice of $\mathbb{T}$, and let $A$ be an affine algebra over $\mathbf{k}$. Defining an algebraic action of $\mathbb{T}$ on $X=\operatorname{Spec} A$ is the same as defining an $M$-grading of $A$. The complexity of the affine $M$-graded algebra $A$ is the codimension of a generic $\mathbb{T}$-orbit in $X$. Note that the classical toric case corresponds to the complexity 0 case.

Let $A$ be a domain and let $I$ be an ideal of $A$. An element $a \in A$ is said to be integral over $I$ if there exist $r \in \mathbb{Z}_{>0}$ and $c_{i} \in I^{i}, i=1, \ldots, r$ such that $a^{r}+\sum_{i=1}^{r} c_{i} a^{r-i}=0$. The integral closure $\bar{I}$ of the ideal $I$ is the set of all elements of $A$ that are integral over $I$. It is known that $\bar{I}$ is an ideal [HS, 1.3.1]. An ideal $I$ is integrally closed if $I=\bar{I}$. Furthermore, $I$ is said to be normal if for any positive integer $e$, the ideal $I^{e}$ is integrally closed. If $A$ is normal then this latter condition is equivalent to the normality of the Rees algebra $A[I t]=A \oplus \bigoplus_{i \geq 1} I^{i} t^{i}$. See [Ri] for more details.

The purpose of this paper is to answer the following questions : given an affine $M$-graded algebra $A$ of complexity 1 over $\mathbf{k}$ and homogeneous elements $a_{1}, \ldots, a_{r} \in A$ such that

$$
A=\mathbf{k}\left[a_{1}, \ldots, a_{r}\right]
$$

can one describe explicitly the normalization of $A$ in terms of the generators $a_{1}, \ldots, a_{r}$ ? Furthermore if $A$ is normal and if $I$ is a homogenous ideal in $A$, can one describe effectively the integral closure of $I$ in terms of a given finite system of homogeneous generators of $I$ ? There exists a connection between these two questions. Indeed the answer to the second can be deduced from that to the first by examining the normalization of the Rees algebra $A[I t]$ corresponding to $I$.

To answer the first question, it is useful to attach an appropriate combinatorial object to a given normal $M$-graded algebra $A$. For instance, in the monomial case if $A$ is normal then the rational cone $\omega$ allows us to reconstruct $A$.

Recall that a $\mathbb{T}$-variety is a normal variety endowed with an effective algebraic $\mathbb{T}$-action. There exists several combinatorial descriptions of affine $\mathbb{T}$-varieties. See [De], [AH] for arbitrary complexity, [KKMS], [Ti] for complexity 1 and [FZ] for the case of surfaces. Note that the description of [AH] is generalized in [AHS] for non-affine $\mathbb{T}$-varieties. See also the survey article [AOPSV] for applications of the theory of $\mathbb{T}$-varieties.

In this paper, we use the point of view of $[\mathrm{AH}]$ and [Ti]. To simplify things we assume that $M=\mathbb{Z}^{n}$ and $\mathbb{T}=\left(\mathbf{k}^{\star}\right)^{n}$. An affine $\mathbb{T}$-variety $X=\operatorname{Spec} A$ of complexity 1 can be described by its weight cone $\omega \subset \mathbb{Q}^{n}$ and by a polyhedral divisor $\mathfrak{D}$ on a smooth algebraic curve $C$, whose coefficients are polyhedra in $\mathbb{Q}^{n}$. For 
any element $m=\left(m_{1}, \ldots, m_{n}\right)$ of $\omega \cap \mathbb{Z}^{n}$, we have an evaluation $\mathfrak{D}(m)$ belonging to the $\mathbb{Q}$-linear space of rational Weil divisors on $C$. Given a combinatorial data $(\omega, C, \mathfrak{D})$ one can construct an $M$-graded algebra

$$
A[C, \mathfrak{D}]:=\bigoplus_{m \in \omega \cap M} H^{0}\left(C, \mathcal{O}_{C}(\lfloor\mathfrak{D}(m)\rfloor)\right) \chi^{m}
$$

where $\chi^{m}$ is the Laurent monomial $t_{1}^{m_{1}} \ldots t_{n}^{m_{n}}$. See [AH] for definitions and specific statements. One of the main results of this paper can be stated as follows (see Theorem 2.4).

Theorem. Let $C$ be a smooth algebraic curve. Consider a subalgebra

$$
B=\mathbf{k}[C]\left[f_{1} \chi^{s_{1}}, \ldots, f_{r} \chi^{s_{r}}\right] \subset L_{[n]}(\mathbf{k}(C)):=\mathbf{k}(C)\left[t_{1}, t_{1}^{-1}, \ldots, t_{n}, t_{n}^{-1}\right]
$$

such that

$$
\operatorname{Frac} B=\operatorname{Frac} L_{[n]}(\mathbf{k}(C)),
$$

where $s_{i} \in \mathbb{Z}^{n}$, $\chi^{s_{i}}$ is the corresponding Laurent monomial, and $f_{i} \in \mathbf{k}(C)^{\star}$. Then the normalization of $B$ is the algebra $A[C, \mathfrak{D}]$ with its weight cone $\omega$ generated by $s_{1}, \ldots, s_{r}$ and with the following coefficient of the polyhedral divisor $\mathfrak{D}$ at the point $z \in C$ :

$$
\Delta_{z}=\left\{v \in \mathbb{Q}^{n},\left\langle s_{i}, v\right\rangle \geq-\nu_{z}\left(f_{i}\right), i=1, \ldots, r\right\},
$$

where $\nu_{z}\left(f_{i}\right)$ is the order of $f_{i}$ at $z$.

This theorem answers the first question. It generalizes well known results for the case of affine surfaces with $\mathbf{k}^{\star}$-actions $[\mathrm{FZ}, 3.9,4.6]$. Note also that it may be applied

$\left(^{*}\right)$ to find a proper polyhedral divisor representing a (normal) subalgebra given by generators;

$(* *)$ to find generators of an algebra represented by a proper polyhedral divisor : the idea is to guess some generating set and apply $(*)$.

The answer to the second question is given by Theorem 4.2. It is known that the set of integrally closed homogeneous ideals of the affine toric variety with weight cone $\omega$ is in bijective correspondence with the set of integral $\omega$-polyhedra contained in $\omega$ (see [KKMS] and section 3). We provide a similar correspondence for integrally closed homogeneous ideals on affine $\mathbb{T}$-varieties of complexity 1 (see Theorem 4.6) that is totally combinatorial when $C$ is affine (see Corollary 4.7).

Any integrally closed homogeneous ideal $I$ of an affine $\mathbb{T}$-variety $X=\operatorname{Spec} A$ of complexity 1 can be described by means of a pair $(P, \widetilde{\mathfrak{D}})$ where $P$ is an integral polyhedron in $\mathbb{Q}^{n}$. This polyhedron plays the same role as the Newton polyhedron does in the monomial case. The polyhedral divisor $\widetilde{\mathfrak{D}}$ corresponds to the normalization of the Rees algebra of $I$. We give a geometric interpretation of the coefficients of $\widetilde{\mathfrak{D}}$. Assume for instance that the weight cone of $A$ is strongly convex, and let $\widetilde{\Delta}_{z}$ be the coefficient of $\widetilde{\mathfrak{D}}$ at a point $z \in C$. Then Theorem 4.6 provides conditions on the equations of facets of $\widetilde{\Delta}_{z}$ so that $\widetilde{\mathfrak{D}}$ corresponds to the normalization of the Rees algebra $A[I t]$.

We provide as well sufficient conditions on $(P, \widetilde{\mathfrak{D}})$ in order that $I$ be normal (see Theorem 5.3). For the case of non-elliptic affine $\mathbf{k}^{\star}$-surfaces, we obtain a combinatorial proof for the normality of any integrally closed invariant ideals of such surfaces. As another application, we obtain the following new criterion of normality which generalizes Reid-Roberts-Vitulli's Theorem [RRV, 3.1] in the case of complexity 0 (see $5.5)$. 
Theorem. Let $n \geq 1$ be an integer and let $\mathbf{k}^{[n+1]}=\mathbf{k}\left[x_{0}, \ldots, x_{n}\right]$ be the algebra of polynomials in $n+1$ variables over $\mathbf{k}$. We endow $\mathbf{k}^{[n+1]}$ with the $\mathbb{Z}^{n}$-grading given by

$$
\mathbf{k}^{[n+1]}=\bigoplus_{m=\left(m_{1}, \ldots, m_{n}\right) \in \mathbb{N}^{n}} A_{m} \text {, where } A_{m}=\mathbf{k}\left[x_{0}\right] x_{1}^{m_{1}} \ldots x_{n}^{m_{n}} .
$$

For a homogeneous ideal I of $\mathbf{k}^{[n+1]}$ the following are equivalent.

(i) The ideal I is normal;

(ii) For any $e \in\{1, \ldots, n\}$, the ideal $I^{e}$ is integrally closed.

Let us give a brief summary of the contents of each section. In the first section, we recall some notions on tori actions of complexity 1 and on polyhedral divisors of Altmann-Hausen. In the second section, we treat the normalization problem for multigraded algebras and show Theorem 2.4. The third section focuses on integrally closed monomial ideals. In section 4 , we study the description by the pair $(P, \widetilde{\mathfrak{D}})$ for integrally closed homogeneous ideals of affine $\mathbb{T}$-varieties. Finally, in the last section, we discuss the problem of normality in a special class.

Throughout this paper $\mathbf{k}$ is an algebraically closed field of characteristic zero. By a variety we mean an integral separated scheme of finite type over $\mathbf{k}$.

Acknowledgments : The author is grateful to Mikhail Zaidenberg for his permanent encouragement and for posing the problem of section 2. We thank Ronan Terpereau and Mateusz Michalek for useful discussions. We thank also the referees for pertinent remarks that allowed to improve the presentation.

\section{T-variétés affines de complexité un et géométrie convexe}

Nous rappelons ici les notions basiques sur les opérations de tores algébriques dont nous aurons besoin par la suite.

1.1. Soit $N$ un réseau de $\operatorname{rang} n$ et $M=\operatorname{Hom}(N, \mathbb{Z})$ son réseau dual. On note $N_{\mathbb{Q}}=\mathbb{Q} \otimes_{\mathbb{Z}} N$ et $M_{\mathbb{Q}}=\mathbb{Q} \otimes_{\mathbb{Z}} M$ les $\mathbb{Q}$-espaces vectoriels duaux associés. Au réseau $M$, on associe un tore algébrique $\mathbb{T}$ de dimension $n$ dont son algèbre des fonctions régulières est définie par

$$
\mathbf{k}[\mathbb{T}]=\bigoplus_{m \in M} \mathbf{k} \chi^{m}
$$

La famille $\left(\chi^{m}\right)_{m \in M}$ satisfaisant les relations $\chi^{m} \cdot \chi^{m^{\prime}}=\chi^{m+m^{\prime}}$, pour tous $m, m^{\prime} \in M$. Le choix d'une base de $M$ donne un isomorphisme entre $\mathbf{k}[\mathbb{T}]$ et l'algèbre des polynômes de Laurent à $n$ variables. Chaque fonction $\chi^{m}$ s'interprète comme un caractère de $\mathbb{T}$.

1.2. Soit $X$ une variété affine et supposons que $\mathbb{T}$ opère algébriquement dans $X$. Alors cela induit une opération de $\mathbb{T}$ dans $A:=\mathbf{k}[X]$ définie par $(t \cdot f)(x)=f(t \cdot x)$ avec $t \in \mathbb{T}, f \in \mathbf{k}[X]$ et $x \in X$, faisant du k-espace vectoriel $A$ un $\mathbb{T}$-module rationnel. Le $\mathbb{T}$-module $A$ admet une décomposition $A=\bigoplus_{m \in M} A_{m}$ en somme directe de sous-espaces vectoriels où pour tout $m \in M$,

$$
A_{m}=\left\{f \in A \mid \forall t \in \mathbb{T}, t \cdot f=\chi^{m}(t) f\right\} .
$$


L'algèbre $A$ est ainsi munie d'une $M$-graduation. Réciproquement, toute $M$-graduation de la k-algèbre $A$ est obtenue par une opération algébrique de $\mathbb{T}$ dans $X=\operatorname{Spec} A$. La partie

$$
S:=\left\{m \in M \mid A_{m} \neq\{0\}\right\}
$$

de $M$ contenant 0 et stable par l'addition est appelée semi-groupe des poids de $A$. Puisque $A$ est de type fini sur k, l'ensemble $S$ engendre un cône polyédral $\omega \subset M_{\mathbb{Q}}$ dit cône des poids de $A$.

L'opération de $\mathbb{T}$ dans $X$ est fidèle si et seulement si $S$ n'est pas contenu dans un sous-réseau propre de $M$. Dans ce cas, le cône $\omega$ est de dimension $n$ et il existe un unique cône polyédral saillant $\sigma \subset N_{\mathbb{Q}}$ tel que

$$
\omega=\left\{m \in M_{\mathbb{Q}} \mid \forall v \in \sigma, m(v) \geq 0\right\} .
$$

On dit que $\omega$ est le cône dual de $\sigma$. On le note $\sigma^{\vee}$. Une $\mathbb{T}$-variété est une variété normale munie d'une opération algébrique fidèle de $\mathbb{T}$.

1.3. Nous appelons complexité de l'opération de $\mathbb{T}$ dans $X=\operatorname{Spec} A$, le degré de transcendance de l'extension de corps $\mathbf{k}(X)^{\mathbb{T}} / \mathbf{k}$. La complexité s'interprète géométriquement comme la codimension d'une orbite en position générale. Lorsque l'opération est fidèle, elle est égale à $\operatorname{dim} X-\operatorname{dim} \mathbb{T}$. Elle fut introduite dans $[\mathrm{LV}]$ pour les opérations de groupes algébriques réductifs dans les espaces homogènes.

Les $\mathbb{T}$-variétés affines de complexité 0 sont les variétés toriques affines. Elles admettent une description bien connue en terme de cônes polyédraux saillants. Pour plus d'informations, voir [CLS], [Da], [Fu], ainsi que $[\mathrm{Od}]$.

Etant donnés un sous-semi-groupe $E \subset M$ et un corps $K_{0}$, nous notons

$$
K_{0}[E]=\bigoplus_{m \in E} K_{0} \chi^{m}
$$

l'algèbre du semi-groupe $E$ sur le corps $K_{0}$. Pour tout cône polyédral $\tau \subset M_{\mathbb{Q}}$, nous écrivons $\tau_{M}:=\tau \cap M$.

Nous rappelons une description combinatoire des $\mathbb{T}$-variétés affines de complexité 1 obtenue dans [AH], [Ti]. Voir [FZ] pour la présentation de Dolgachev-Pinkham-Demazure (D.P.D.) des $\mathbb{C}^{\star}$-surfaces affines complexes. Notons que l'approche donnée dans [Ti] provient d'une description antérieure des opérations de groupes algébriques réductifs de complexité 1 [Ti 2]. Dans [Vo], on donne un lien entre [AH] et la description classique de [KKMS].

1.4. Soit $C$ une courbe algébrique lisse et $\sigma \subset N_{\mathbb{Q}}$ un cône polyédral saillant. Une partie $\Delta \subset N_{\mathbb{Q}}$ est un $\sigma$-polyèdre si $\Delta$ est somme de Minkowski de $\sigma$ et d'un polytope $Q \subset N_{\mathbb{Q}}$. On note $\operatorname{Pol}_{\sigma}\left(N_{\mathbb{Q}}\right)$ le semi-groupe abélien des $\sigma$-polyèdres de loi la somme de Minkowski et d'élément neutre $\sigma$.

Pour $\Delta \in \operatorname{Pol}_{\sigma}\left(N_{\mathbb{Q}}\right)$, on définit la fonction $h_{\Delta}: \sigma^{\vee} \rightarrow \mathbb{Q}$ par $h_{\Delta}(m)=\min _{v \in \Delta} m(v)$, pour tout $m \in \sigma^{\vee}$. On dit que $h_{\Delta}$ est la fonction de support de $\Delta$. Elle est identiquement nulle si et seulement si $\Delta=\sigma$. Pour tous $m, m^{\prime} \in \sigma^{\vee}$, on a l'inégalité de sous-additivité

$$
h_{\Delta}(m)+h_{\Delta}\left(m^{\prime}\right) \leq h_{\Delta}\left(m+m^{\prime}\right) .
$$

Un diviseur $\sigma$-polyédral sur $C$ est une somme formelle

$$
\mathfrak{D}=\sum_{z \in C} \Delta_{z} \cdot z
$$


où chaque $\Delta_{z}$ appartient à $\operatorname{Pol}_{\sigma}\left(N_{\mathbb{Q}}\right)$ avec pour presque1 11 tout $z \in C, \Delta_{z}=\sigma$. On note $\operatorname{supp}(\mathfrak{D})$, dit support de $\mathfrak{D}$, l'ensemble des points $z \in C$ tels que $\Delta_{z} \neq \sigma$. On dit que $\mathfrak{D}$ est propre lorsque pour tout $m \in \sigma_{M}^{\vee}$, l'évaluation

$$
\mathfrak{D}(m):=\sum_{z \in C} h_{\Delta_{z}}(m) \cdot z
$$

est un $\mathbb{Q}$-diviseur semi-ample et abondant (big) pour $m$ appartenant à l'intérieur relatif de $\sigma^{\vee}$.

1.5. La propreté de $\mathfrak{D}$ est décrite en distinguant les deux cas suivants [AH, 2.12].

(i) Lorsque $C$ est une courbe projective lisse, le diviseur polyédral $\mathfrak{D}$ est propre si et seulement si pour tout vecteur $m \in \sigma_{M}^{\vee}$, on a $\operatorname{deg}(\mathfrak{D}(m)) \geq 0$ et si $\operatorname{deg}(\mathfrak{D}(m))=0$ alors $m$ appartient au bord de $\sigma^{\vee}$ et un multiple entier non nul de $\mathfrak{D}(m)$ est principal;

(ii) Lorsque $C$ est une courbe affine lisse, aucune condition n'est imposée sur l'évaluation de $\mathfrak{D}$.

Notation 1.6. D'après l'inégalité (1) dans 1.4 , si $\mathfrak{D}$ est un diviseur $\sigma$-polyédral sur $C$ alors pour tous vecteurs $m, m^{\prime} \in \sigma_{M}^{\vee}$, l'application

$$
H^{0}\left(C, \mathcal{O}_{C}(\lfloor\mathfrak{D}(m)\rfloor)\right) \times H^{0}\left(C, \mathcal{O}_{C}\left(\left\lfloor\mathfrak{D}\left(m^{\prime}\right)\right\rfloor\right)\right) \rightarrow H^{0}\left(C, \mathcal{O}_{C}\left(\left\lfloor\mathfrak{D}\left(m+m^{\prime}\right)\right\rfloor\right)\right), \quad(f, g) \mapsto f \cdot g
$$

est bien définie. Le $\mathbb{T}$-module

$$
A[C, \mathfrak{D}]:=\bigoplus_{m \in \sigma_{M}^{\vee}} H^{0}\left(C, \mathcal{O}_{C}(\lfloor\mathfrak{D}(m)\rfloor)\right) \chi^{m}
$$

est donc une algèbre $M$-graduée. On la notera $A[\mathfrak{D}]$ lorsque la mention de $C$ est claire. Pour tout élément homogène $f \chi^{m}$ de $\mathbf{k}(C)[M]$, nous sous-entendons que $f \in k(C)^{\star}$ et que $\chi^{m}$ est un caractère du tore $\mathbb{T}$.

Le théorème suivant décrit les algèbres affines normales $M$-graduées de complexité 1 et de cône des poids $\sigma^{\vee}$ (voir $\left.[\mathrm{AH}],[\mathrm{Ti}]\right)$.

Théorème 1.7. $\quad$ (i) $S i \mathfrak{D}$ est un diviseur $\sigma$-polyédral propre sur une courbe algébrique lisse $C$ alors l'algèbre multigraduée $A=A[C, \mathfrak{D}]$ est normale, de type fini sur $\mathbf{k}$ et sa $M$-graduation fait de $X=\operatorname{Spec} A$ une $\mathbb{T}$-variété de complexité 1 . Réciproquement, toute algèbre des fonctions régulières d'une $\mathbb{T}$-variété affine de complexité 1 est isomorphe comme algèbre $M$-graduée à $A[C, \mathfrak{D}]$, où $C$ est une courbe algébrique lisse et $\mathfrak{D}$ est un diviseur polyédral propre sur $C$.

(ii) Plus précisément, toute sous-algèbre multigraduée $A \subset \mathbf{k}(C)[M]$ normale, de type fini sur $\mathbf{k}$, satisfaisant $A_{0}=\mathbf{k}[C]$, de même corps des fractions que $A$ et ayant $\sigma^{\vee}$ pour cône des poids, est égale à $A[C, \mathfrak{D}]$ pour un unique diviseur $\sigma$-polyédral $\mathfrak{D}$ propre sur $C$.

Notation 1.8. Si $\mathfrak{D}$ est un diviseur polyédral propre sur $C$ alors on note par $X[C, \mathfrak{D}]$ la $\mathbb{T}$-variété affine Spec $A[C, \mathfrak{D}]$ correspondante.

1. Cela signifie qu'il existe un sous-ensemble fini $F \subset C$ tel que pour tout $z \in C-F, \Delta_{z}=\sigma$. 
L'assertion suivante est connue [AH, §8], [Li, Theorem 1.4(3)]. Elle permet de comparer deux données combinatoires $(C, \sigma, \mathfrak{D})$ et $\left(C^{\prime}, \sigma^{\prime}, \mathfrak{D}^{\prime}\right)$ donnant des k-algèbres $M$-graduées isomorphes.

Théorème 1.9. Soient $C, C^{\prime}$ des courbes algébriques lisses et $\mathfrak{D}, \mathfrak{D}^{\prime}$ des diviseurs polyédraux propres respectivement sur $C, C^{\prime}$ selon des cônes polyédraux saillants $\sigma, \sigma^{\prime} \subset N_{\mathbb{Q}}$. Alors $X[C, \mathfrak{D}]$ et $X\left[C^{\prime}, \mathfrak{D}^{\prime}\right]$ sont $\mathbb{T}$-isomorphes si et seulement si il existe un automorphisme de réseau $F: N \rightarrow N$ tel que $F_{\mathbb{Q}}(\sigma)=\sigma^{\prime}$, un isomorphisme $\phi: C \rightarrow C^{\prime}$ de courbes algébriques, $v_{1}, \ldots, v_{r} \in N$ et $f_{1}, \ldots, f_{r} \in k(C)^{\star}$ des fonctions rationnelles non nulles tels que pour tout $m \in \sigma_{M}^{\prime \vee}$,

$$
\phi^{\star}\left(\mathfrak{D}^{\prime}\right)(m)=F_{\star}(\mathfrak{D})(m)+\sum_{i=1}^{r} m\left(v_{i}\right) \cdot \operatorname{div}\left(f_{i}\right)
$$

avec

$$
\mathfrak{D}=\sum_{z \in C} \Delta_{z} \cdot z, \mathfrak{D}^{\prime}=\sum_{z^{\prime} \in C^{\prime}} \Delta_{z^{\prime}}^{\prime} \cdot z^{\prime}, \phi^{\star}\left(\mathfrak{D}^{\prime}\right)=\sum_{z^{\prime} \in C^{\prime}} \Delta_{z^{\prime}}^{\prime} \cdot \phi^{-1}\left(z^{\prime}\right) \text { et } F_{\star}(\mathfrak{D})=\sum_{z \in C}\left(F_{\mathbb{Q}}\left(\Delta_{z}\right)+\sigma\right) \cdot z .
$$

La proposition suivante montre que la fonction de support d'un $\sigma$-polyèdre est linéaire par morçeaux. La preuve de ce résultat est aisée et laissée aux lecteurs.

Lemme 1.10. Soit $\sigma \subset N_{\mathbb{Q}}$ un cône polyédral saillant, $\Delta \in \operatorname{Pol}_{\sigma}\left(N_{\mathbb{Q}}\right)$ un polyèdre et $S \subset \Delta$ une partie non vide. Notons $V(\Delta)$ l'ensemble des sommets de $\Delta$. Alors $\Delta=\operatorname{Conv}(S)+\sigma$ si et seulement si pour tout $m \in \sigma^{\vee}, h_{\Delta}(m)=\min _{v \in S} m(v)$. En particulier, pour tout $m \in \sigma^{\vee}$, on a $h_{\Delta}(m)=\min _{v \in V(\Delta)} m(v)$.

La terminologie suivante est classique pour les $\mathbb{C}^{\star}$-surfaces affines complexes [FZ]. Elle est introduite dans $[\mathrm{Li}]$ pour les $\mathbb{T}$-variétés affines de complexité 1.

Définition 1.11. Soit $X$ une variété affine. Une opération algébrique de $\mathbb{T}$ de complexité 1 dans $X$ est dite elliptique si l'algèbre multigraduée correspondante

$$
A=\mathbf{k}[X]=\bigoplus_{m \in M} A_{m} \text { avec } A_{m}=\left\{f \in A \mid \forall t \in \mathbb{T}, t \cdot f=\chi^{m}(t) f\right\}
$$

vérifie $A_{0}=\mathbf{k}$. Dans ce cas, on dit que l'algèbre $M$-graduée $A$ est elliptique.

Remarque 1.12. Considérons $A=A[C, \mathfrak{D}]$. Alors $A$ est elliptique si et seulement si $C$ est projective. L'ellipticité de $A$ donne des contraintes géométriques sur la variété $X=\operatorname{Spec} A$. En effet, si $C$ est affine alors $X$ est toroïdale [KKMS] et n'admet donc que des singularités toriques. Tandis que lorsque $C$ est projective de genre $g \geq 1$, la variété $X$ a au moins une singularité qui n'est pas rationnelle [LS, Propositions $5.1,5.5]$.

La proposition suivante peut être vue comme une généralisation de [FZ, Lemma 1.3(a)].

Proposition 1.13. Soit $X$ une $\mathbb{T}$-variété affine de complexité 1 et notons $\sigma \subset N_{\mathbb{Q}}$ le dual du cône des poids de l'algèbre multigraduée

$$
A=\mathbf{k}[X]=\bigoplus_{m \in M} A_{m} \text { avec } A_{m}=\left\{f \in A \mid \forall t \in T, t \cdot f=\chi^{m}(t) f\right\}
$$

obtenue par l'opération de $\mathbb{T}$. Les assertions suivantes sont vraies.

2. Un automorphisme de réseau $F: N \rightarrow N$ induit un automorphisme de l'espace vectoriel $N_{\mathbb{Q}}$ que l'on note $F_{\mathbb{Q}}$. 
(i) Si l'opération de $\mathbb{T}$ n'est pas elliptique alors le semi-groupe des poids de $\mathbf{k}[X]$ est $\sigma_{M}^{\vee}$ et pour tout $m \in \sigma_{M}^{\vee}$, le $A_{0}$-module $A_{m}$ est localement libre de rang 1 ;

(ii) Si l'opération est elliptique alors $\sigma \neq\{0\}$;

(iii) L'intersection du sous-ensemble

$$
L=\left\{m \in \sigma_{M}^{\vee} \mid A_{m}=\{0\}\right\} \subset M_{\mathbb{Q}}
$$

avec toute droite vectorielle rencontrant l'intérieur relatif de $\sigma^{\vee}$ est finie.

Démonstration. Si l'opération de $\mathbb{T}$ n'est pas elliptique alors par le théorème 1.7, on peut supposer qu'il existe une courbe algébrique affine lisse $C$ et un diviseur $\sigma$-polyédral propre $\mathfrak{D} \operatorname{sur} C$ tels que pour tout $m \in \sigma_{M}^{\vee}$,

$$
A_{m}=H^{0}\left(C, \mathcal{O}_{C}(\lfloor\mathfrak{D}(m)\rfloor)\right) \chi^{m} \text { et } \mathbf{k}[X]=\bigoplus_{m \in \sigma_{M}^{\vee}} A_{m}
$$

Comme $C$ est affine, pour tout $m \in \sigma_{M}^{\vee}$, on a $A_{m} \neq\{0\}$. D'où l'assertion (i).

Si l'opération est elliptique alors on peut supposer que $\mathbf{k}[X]$ vérifie $(2)$ avec $C$ une courbe projective lisse de genre $g$. Si $\sigma=\{0\}$ alors $\sigma^{\vee}=M_{\mathbb{Q}}$. Pour tout $m \in \sigma_{M}^{\vee}$, on a par propreté $: \operatorname{deg}(\mathfrak{D}(m))>0$. L'égalité $\mathfrak{D}(0)=0$ donne une contradiction. D'où l'assertion (ii).

Soit $m \in \sigma_{M}^{\vee}$ appartenant à l'intérieur relatif de $\sigma^{\vee}$. Alors il existe $r_{0} \in \mathbb{Z}_{>0}$ tel que pour tout $z \in \operatorname{supp}(\mathfrak{D})$ et tout sommet $v$ de $\Delta_{z}, r_{0} v \in N$. Ainsi, par le lemme 1.10,

$$
\left.\mathfrak{D}\left(r_{0} m\right)=\left\lfloor\mathfrak{D}\left(r_{0} m\right)\right)\right\rfloor .
$$

Par la propreté de $\mathfrak{D}$, on peut supposer que

$$
\operatorname{deg}\left(\mathfrak{D}\left(r_{0} m\right)\right)=\operatorname{deg}\left(\left\lfloor\mathfrak{D}\left(r_{0} m\right)\right\rfloor\right)>d+g-1
$$

où $d$ est le cardinal de $\operatorname{supp}(\mathfrak{D})$. Donc pour tout $r \in \mathbb{N}$,

$$
\operatorname{deg}\left(\left\lfloor\mathfrak{D}\left(\left(r_{0}+r\right) m\right)\right\rfloor\right)=\operatorname{deg}\left(\left\lfloor\mathfrak{D}\left(r_{0} m\right)\right\rfloor\right)+\operatorname{deg}(\lfloor\mathfrak{D}(r m)\rfloor) \geq \operatorname{deg}\left(\left\lfloor\mathfrak{D}\left(r_{0} m\right)\right\rfloor\right)-d>g-1 .
$$

Comme $\sigma \neq\{0\}$, la demi-droite $\mathbb{Q}_{\leq 0} \cdot m$ ne rencontre $\sigma^{\vee}$ qu'en l'origine [CLS, Exercice 1.2.4]. Par le théorème de Riemann-Roch, (4) donne l'inclusion

$$
\mathbb{Q} \cdot m \cap L \subset\left\{0, m, 2 m, \ldots,\left(r_{0}-1\right) m\right\} .
$$

D'où le résultat.

Remarque 1.14. Notons $\sigma=\mathbb{Q}_{\geq 0}^{2}$. L'exemple du diviseur polyédral propre

$\mathfrak{D}=\Delta_{0} \cdot\{0\}+\Delta_{1} \cdot\{1\}+\Delta_{\infty} \cdot\{\infty\}, \Delta_{0}=\left(\frac{1}{2}, 0\right)+\sigma, \Delta_{1}=\left(-\frac{1}{2}, 0\right)+\sigma, \Delta_{\infty}=[(1,0),(0,1)]+\sigma$

sur $\mathbb{P}^{1}$ montre qu'en général, il existe des demi-droites vectorielles contenues dans le bord de $\sigma^{\vee}$ qui rencontrent $L$ avec une infinité de points. En effet, dans cet exemple, pour tout $r \in \mathbb{N}$, on a

$$
H^{0}\left(\mathbb{P}^{1}, \mathcal{O}_{\mathbb{P}^{1}}(\lfloor\mathfrak{D}(2 r+1,0)\rfloor)\right) \chi^{(2 r+1,0)}=\{0\} .
$$




\section{Normalisation des algèbres affines multigraduées de complexité un}

Le but de cette section est de décrire explicitement la normalisation des algèbres affines multigraduées de complexité 1.

Dans le cas de la complexité 0 , toute algèbre affine $M$-graduée est réalisée comme sous-algèbre $\mathbb{T}$-stable de $\mathbf{k}[M]$. La normalisation de $A$ est déterminée par son cône des poids [Ho]. Par analogie, toute algèbre affine $M$-graduée de complexité 1 est plongée dans une algèbre $\mathbf{k}(C)[M]$ où $C$ est une courbe algébrique lisse. Nous rappelons ceci dans le paragraphe suivant.

2.1. Soit $A=\bigoplus_{m \in M} A_{m}$ une algèbre intègre $M$-graduée de type fini sur $\mathbf{k}$. Notons $K$ son corps des fractions. On suppose que tr.deg $K_{\mathbf{k}} K^{\mathbb{T}}=1$. Sans perte de généralité, on peut supposer que la $M$-graduation de $A$ est fidèle. Alors pour tout vecteur $m \in M$,

$$
K_{m}:=\left\{f / g \mid f \in A_{m+e}, g \in A_{e}, g \neq 0\right\} \subset K
$$

est un sous-espace vectoriel de dimension 1 sur $K_{0}=K^{\mathbb{T}}$. Le choix d'une base de $M$ nous permet de construire une famille $\left(\chi^{m}\right)_{m \in M}$ d'éléments de $K^{\star}$ vérifiant pour tous $m, m^{\prime} \in M$,

$$
K_{m}=K_{0} \chi^{m} \text { et } \chi^{m} \cdot \chi^{m^{\prime}}=\chi^{m+m^{\prime}}
$$

Quitte à remplacer $A_{0}$ par $\overline{A_{0}}$, on peut supposer que $A_{0}$ est normale. Notons que l'algèbre $A_{0}$ est de type fini sur $\mathbf{k}$. Soit $\widetilde{C}$ la courbe complète lisse sur $\mathbf{k}$ obtenue à partir de l'ensemble des valuations discrètes de $K_{0} / \mathbf{k}$, de sorte que $K_{0}=\mathbf{k}(\widetilde{C})$.

Si l'opération de $\mathbb{T}$ dans $X=\operatorname{Spec} A$ n'est pas elliptique alors $K_{0}$ est le corps des fractions de $A_{0}$. En effet, si $b \in K_{0}$ alors $b$ est un élément algébrique sur Frac $A_{0}$. Il existe donc $a \in A_{0}$ non nul tel que $a b$ soit entier sur $A_{0}$. Par normalité de $A_{0}$, on a $a b \in \bar{A} \cap K_{0}=A_{0}$ et donc $K_{0} \subset$ Frac $A_{0}$. L'inclusion réciproque est immédiate. Dans tous les cas, il existe un unique ouvert non vide $C \subset \widetilde{C}$ tel que

$$
A_{0}=\mathbf{k}[C] \text { et } A \subset \bigoplus_{m \in M} K_{m}=\mathbf{k}(C)[M]
$$

Par le théorème 1.7, l'égalité $K=\operatorname{Frac} \mathbf{k}(C)[M]$ implique que $\bar{A}=A[C, \mathfrak{D}]$, pour un unique diviseur polyédral propre $\mathfrak{D}=\sum_{z \in C} \Delta_{z} \cdot z$.

Fixons un système de générateurs homogènes

$$
A=\mathbf{k}[C]\left[f_{1} \chi^{m_{1}}, \ldots, f_{r} \chi^{m_{r}}\right]
$$

avec $f_{1}, \ldots, f_{r}$ des fonctions rationnelles non nulles sur $C$ et $m_{1}, \ldots, m_{r}$ des éléments de $M$. Il s'agit de déterminer les polyèdres $\Delta_{z}$ en fonction de $\left(\left(f_{1}, m_{1}\right), \ldots,\left(f_{r}, m_{r}\right)\right)$. Dans [FZ], on donne la présentation D.P.D. de $\bar{A}$ pour le cas des surfaces affines complexes avec opérations paraboliques et hyperboliques de $\mathbb{C}^{\star}$. Nous rappelons ces résultats dans le corollaire 2.7 .

Le lemme élémentaire suivant sera utilisé dans la preuve du théorème 2.4. La démonstration de ce résultat est laissée aux lecteurs.

Lemme 2.2. Soit $\sigma \subset N_{\mathbb{Q}}$ un cône polyédral saillant et $\Delta_{1}, \Delta_{2} \in \operatorname{Pol}_{\sigma}\left(N_{\mathbb{Q}}\right)$ des $\sigma$-polyèdres. Alors $\Delta_{1}=\Delta_{2}$ si et seulement si pour tout $m \in \sigma_{M}^{\vee}$ appartenant à l'intérieur relatif de $\sigma^{\vee}, h_{\Delta_{1}}(m)=h_{\Delta_{2}}(m)$. 
Notation 2.3. Soient $C$ une courbe algébrique lisse et $f=\left(f_{1} \chi^{m_{1}}, \ldots, f_{r} \chi^{m_{r}}\right)$ un $r$-uplet d'éléments homogènes de $\mathbf{k}(C)[M]$ tels que $\sum_{i=1}^{r} \mathbb{Q} m_{i}=M_{\mathbb{Q}}$. Posons $\sigma \subset N_{\mathbb{Q}}$ le cône polyédral saillant dual de $\sum_{i=1}^{r} \mathbb{Q}_{\geq 0} m_{i}$ et pour tout $z \in C$, considérons $\Delta_{z}[f] \subset N_{\mathbb{Q}}$ le $\sigma$-polyèdre défini par les inégalités

$$
m_{i} \geq-\nu_{z}\left(f_{i}\right), \quad i=1,2, \ldots, r .
$$

On note $\mathfrak{D}[f]$ le diviseur $\sigma$-polyédral $\sum_{z \in C} \Delta_{z}[f] . z$.

Dans le théorème suivant, nous décrivons la normalisation de l'algèbre $A$ donnée dans 2.1.

Théorème 2.4. Soient $C$ une courbe algébrique lisse et

$$
A=\mathbf{k}[C]\left[f_{1} \chi^{m_{1}}, \ldots, f_{r} \chi^{m_{r}}\right] \subset \mathbf{k}(C)[M]
$$

une sous-algèbre $\mathbb{T}$-stable engendrée par des éléments homogènes $f_{1} \chi^{m_{1}}, \ldots, f_{r} \chi^{m_{r}}$. Supposons que $A$ a le même corps des fractions que $\mathbf{k}(C)[M]$ et soit $\bar{A}$ la normalisation de $A$, vue comme sous-algèbre de $\mathbf{k}(C)[M]$. Considérons $\mathfrak{D}[f]$ défini comme dans 2.3. Alors le dual $\sigma$ du cône polyédral $\sum_{i=1}^{r} \mathbb{Q}_{\geq 0} m_{i}$ est saillant et $\mathfrak{D}[f]$ est l'unique diviseur $\sigma$-polyédral propre sur $C$ vérifiant $\bar{A}=A[C, \mathfrak{D}[f]]$.

Démonstration. D'après [HS, Theorem 2.3.2], la sous-algèbre $\bar{A} \subset \mathbf{k}(C)[M]$ est $M$-graduée. Puisque $A$ a même corps des fractions que $\mathbf{k}(C)[M]$, le cône $\sigma$ est saillant. Par le théorème 1.7, il existe un unique diviseur $\sigma$-polyédral propre $\mathfrak{D}$ sur $C$ tel que $\bar{A}=A[C, \mathfrak{D}]$. Posons $B:=A[C, \mathfrak{D}[f]]$. Alors pour tout $i \in\{1, \ldots, r\}$ et tout $z \in C$, on a l'inégalité

$$
h_{\Delta_{z}[f]}\left(m_{i}\right) \geq-\nu_{z}\left(f_{i}\right)
$$

de sorte que pour tout $i \in\{1, \ldots, r\}$,

$$
\mathfrak{D}[f]\left(m_{i}\right)=\sum_{z \in C} h_{\Delta_{z}[f]}\left(m_{i}\right) \cdot z \geq-\operatorname{div}\left(f_{i}\right) .
$$

On obtient l'inclusion $A \subset B$. Comme $B$ est l'intersection d'anneaux de valuations discrètes de Frac $B / \mathbf{k}$ [De, §2.7], l'algèbre $B$ est normale. D'où $\bar{A}=A[\mathfrak{D}] \subset B$.

Montrons l'égalité $\mathfrak{D}=\mathfrak{D}[f]$. Supposons que $C$ est une courbe algébrique projective lisse de genre $g$. Pour tout $m^{\prime} \in \sigma_{M}^{\vee}$, on a

$$
H^{0}\left(C, \mathcal{O}_{C}\left(\left\lfloor\mathfrak{D}\left(m^{\prime}\right)\right\rfloor\right)\right) \subset H^{0}\left(C, \mathcal{O}_{C}\left(\left\lfloor\mathfrak{D}[f]\left(m^{\prime}\right)\right\rfloor\right)\right) .
$$

Soit $m \in \sigma_{M}^{\vee}$ appartenant à l'intérieur relatif de $\sigma^{\vee}$. Prenons $s \in \mathbb{Z}_{>0}$ tels que $s \mathfrak{D}(m)$ et $s \mathfrak{D}[f](m)$ soient des diviseurs de Weil à coefficients entiers. Alors par (5), par le théorème de Riemann-Roch et puisque $\mathfrak{D}$ est propre, il existe $r_{0} \in \mathbb{Z}_{>0}$ tel que pour tout entier $r \geq r_{0}$,

$$
h^{0}\left(C, \mathcal{O}_{C}(r s \mathfrak{D}[f](m))\right) \geq r \operatorname{deg}(s \mathfrak{D}(m))+1-g>1 .
$$

D'après [Ha, IV, Lemma 1.2], pour tout entier $r \geq r_{0}, \operatorname{deg}(r s \mathfrak{D}[f](m))>0$ et donc $\mathfrak{D}[f](m)$ est semiample. Donc par [AH, Lemma 9.1], on a $\mathfrak{D}[f](m) \geq \mathfrak{D}(m)$. Cette inégalité est aussi vraie lorsque $C$ est une courbe algébrique affine lisse. Revenons à l'hypothèse où $C$ est une courbe algébrique lisse quelconque. Les inégalités

$$
\mathfrak{D}\left(m_{i}\right)+\operatorname{div}\left(f_{i}\right) \geq 0, \quad i=1, \ldots, r,
$$

entraînent que pour tout $z \in C, \Delta_{z} \subset \Delta_{z}[f]$. Donc pour tout $m \in \sigma^{\vee}, \mathfrak{D}(m) \geq \mathfrak{D}[f](m)$. Par le lemme 2.2 , on conclut que $\mathfrak{D}=\mathfrak{D}[f]$. 
L'exemple qui suit illustre comment on peut établir la normalité d'une algèbre donnée à partir d'un système de générateurs.

Exemple 2.5. Soit $z$ une variable et soit $\mathbb{T}$ le tore $\left(\mathbf{k}^{\star}\right)^{2}$. Considérons la sous-algèbre $\mathbb{T}$-stable

$$
A=\mathbf{k}\left[\frac{z}{z-1} \chi^{(2,0)}, \chi^{(0,1)}, z \chi^{(2,2)}, \frac{z^{2}}{z-1} \chi^{(3,2)}\right] \subset \mathbf{k}(z)\left[\mathbb{Z}^{2}\right]
$$

Alors on a les égalités $A^{\mathbb{T}}=\mathbf{k}$ et $(\operatorname{Frac} A)^{\mathbb{T}}=\mathbf{k}(z)$. Le cône des poids $\sigma^{\vee} \subset \mathbb{Q}^{2}$ de $A$ est le quadrant positif. Par le théorème 2.4 , la normalisation $\bar{A}$ de $A$ est égale à $A\left[\mathbb{P}^{1}, \mathfrak{D}\right]$ où $\mathfrak{D}=\Delta_{0}(0)+\Delta_{1}(1)+\Delta_{\infty}(\infty)$ est le diviseur $\sigma$-polyédral propre sur $\mathbb{P}^{1}$ défini par

$$
\Delta_{0}=-\left(\frac{1}{2}, 0\right)+\sigma, \Delta_{1}=\left(\frac{1}{2}, 0\right)+\sigma, \Delta_{\infty}=\left[\left(\frac{1}{2}, 0\right),\left(0, \frac{1}{2}\right)\right]+\sigma .
$$

Par exemple, le polyèdre $\Delta_{0}$ est donné par les inégalités

$$
\begin{gathered}
2 x \geq-\nu_{0}\left(\frac{z}{z-1}\right)=-1, \quad y \geq-\nu_{0}(1)=0,2 x+2 y \geq-\nu_{0}(z)=-1, \\
3 x+2 y \geq-\nu_{0}\left(\frac{z^{2}}{z-1}\right)=-2 .
\end{gathered}
$$

Un calcul direct montre qu'en fait $A=A\left[\mathbb{P}^{1}, \mathfrak{D}\right]$. Si l'on pose

$$
t_{1}:=\frac{z}{z-1} \chi^{(2,0)}, t_{2}:=\chi^{(0,1)}, t_{3}:=z \chi^{(2,2)}, t_{4}:=\frac{z^{2}}{z-1} \chi^{(3,2)},
$$

alors les fonctions $t_{1}, t_{2}, t_{3}, t_{4}$ vérifient la relation irréductible $t_{4}^{2}-t_{1}^{2} t_{2}^{2} t_{3}-t_{1} t_{3}^{2}=0$. On conclut que l'hypersurface $V\left(x_{4}^{2}-x_{1}^{2} x_{2}^{2} x_{3}-x_{1} x_{3}^{2}\right) \subset \mathbb{A}^{4}$ est normale.

Rappelons que pour une variété algébrique affine $X$, on note SAut $X$ le sous-groupe des automorphismes de $X$ engendré par la réunion des images des opérations algébriques de $\mathbb{G}_{a}$ dans $X$. Pour plus d'informations voir [AKZ] et [AFKKZ] où la notion de variété flexible est introduite. L'exemple suivant est inspiré de [LS, Example 1.1]. Il correspond au cas de $d=3$ et de $e=2$.

Exemple 2.6. Soient $d, e \geq 2$ des entiers tels que $d+1=e^{2}$. Notons

$$
\mathfrak{D}_{d, e}=\Delta_{0}^{d, e} \cdot\{0\}+\Delta_{1}^{d, e} \cdot\{1\}+\Delta_{\infty}^{d, e} \cdot\{\infty\}
$$

le diviseur polyédral propre sur $\mathbb{P}^{1}$ avec

$$
\Delta_{0}^{d, e}=[(1,0),(1,1)]+\sigma_{d e}, \Delta_{1}^{d, e}=\left(-\frac{1}{e}, 0\right)+\sigma_{d e}, \Delta_{\infty}^{d, e}=\left(\frac{e}{d}-1,0\right)+\sigma_{d e}
$$

où $\sigma_{d e}$ est le cône de $\mathbb{Q}^{2}$ engendré par les vecteurs $(1,0)$ et $(1, d e)$, et $X_{d, e}:=X\left[\mathfrak{D}_{d, e}\right]$. Alors $X_{d, e}$ n'est pas torique. En effet, $X_{d, e}$ n'est pas $\mathbb{T}$-isomorphe à $X\left[\mathbb{P}^{1}, \mathfrak{D}\right]$ avec $\mathfrak{D}$ un diviseur polyédral propre sur $\mathbb{P}^{1}$ porté par au plus 2 points, i.e. Card $\operatorname{Supp} \mathfrak{D} \leq 2$ (voir [AL, Corollary 1.4] et le théorème 1.9). 
Montrons que le groupe SAut $X_{d, e}$ opère infiniment transitivement dans le lieu lisse de $X_{d, e}$. Pour cela considérons la sous-algèbre

$$
A_{d, e}:=\mathbf{k}\left[\chi^{(0,1)}, \frac{(1-z)^{d}}{z^{d e-1}} \chi^{(d e,-1)}, \frac{1-z}{z^{e}} \chi^{(e, 0)}, \frac{(1-z)^{e}}{z^{d}} \chi^{(d, 0)}\right] \subset \mathbf{k}(z)\left[\mathbb{Z}^{2}\right]
$$

où $\mathbb{T}$ est le tore $\left(\mathbf{k}^{\star}\right)^{2}$ et $z$ est une variable sur $\mathbf{k}(\mathbb{T})$. Posons

$$
u:=\chi^{(0,1)}, \quad v:=\frac{(1-z)^{d}}{z^{d e-1}} \chi^{(d e,-1)}, x:=\frac{1-z}{z^{e}} \chi^{(e, 0)}, \quad y:=\frac{(1-z)^{e}}{z^{d}} \chi^{(d, 0)} .
$$

Alors les fonctions $u, v, x, y$ vérifient la relation irréductible $u v+x^{d}-y^{e}=0(E)$, identifiant $\operatorname{Spec} A_{d, e}$ avec l'hypersurface $H_{d, e}$ d'équation $(E)$ de $\mathbb{A}^{4}$. L'origine $O$ de $\mathbb{A}^{4}$ est l'unique point singulier de $H_{d, e}$.

D'après [Vie, Proposition 2], $H_{d, e}$ est normale en $O$ et par [KZ, §5], [AKZ, Theorem 3.2], le groupe SAut $H_{d, e}$ opère infiniment transitivement dans $H_{d, e}-\{O\}$ comme suspension du plan affine. Par ailleurs, on a $\left(\operatorname{Frac} A_{d, e}\right)^{\mathbb{T}}=\mathbf{k}(z)$ et Frac $A_{d, e}=\operatorname{Frac} \mathbf{k}(z)\left[\mathbb{Z}^{2}\right]$. Un calcul facile montre que $\mathfrak{D}[u, v, x, y]=\mathfrak{D}_{d, e}$. On conclut par le théorème 2.4 .

Notons que le calcul de $\mathfrak{D}_{d, e}$ peut être obtenu à partir de $H_{d, e}$ en utilisant [AH, §11].

Le théorème 2.4 appliqué à $\mathbb{T}=\mathbf{k}^{\star}$ donne le corollaire suivant. Les parties concernant les cas paraboliques et hyperboliques ont été établies dans [FZ, 3.9, 4.6]. Grâce à ces résultats, il est montré dans [FZ] que toute $\mathbb{C}^{\star}$-surface complexe normale affine parabolique ayant un bon quotient isomorphe à $\mathbb{A}_{\mathbb{C}}^{1}$ est la normalisation d'une hypersurface de $\mathbb{A}_{\mathbb{C}}^{3}$ d'équation $x^{d}=y P(z)$, où $P \in \mathbb{C}[z]$ est un polynôme. Une description analogue est donnée dans le cas hyperbolique, c.f. [FZ, 4.8].

Corollaire 2.7. Soit $C$ une courbe algébrique lisse, considérons $\chi$ une variable sur $\mathbf{k}(C)$ et soit $A$ une sousalgèbre graduée de $\mathbf{k}(C)[\mathbb{Z}]$ ayant le même corps des fractions que $\mathbf{k}(C)[\mathbb{Z}]$. Alors les assertions suivantes sont vraies.

(i) (cas elliptique et parabolique) $S i$

$$
A=\mathbf{k}[C]\left[f_{1} \chi^{m_{1}}, \ldots, f_{r} \chi^{m_{r}}\right] \subset \mathbf{k}(C)[\mathbb{Z}],
$$

avec $f_{i} \in \mathbf{k}(C)^{\star}$ et $m_{1}, \ldots, m_{r} \in \mathbb{Z}_{>0}$, alors la présentation de Dolgachev-Pinkham-Demazure (D.P.D.) de $\bar{A}=A_{C, D}$ est donnée par le $\mathbb{Q}$-diviseur sur $C$

$$
D=-\min _{1 \leq i \leq r} \frac{\operatorname{div}\left(f_{i}\right)}{m_{i}}
$$

(ii) (cas hyperbolique) $S i C=\operatorname{Spec} A_{0}$ est une courbe affine lisse,

$$
A=\mathbf{k}[C]\left[f_{1} \chi^{-m_{1}}, \ldots, f_{r} \chi^{-m_{r}}, g_{1} \chi^{n_{1}}, \ldots, g_{s} \chi^{n_{s}}\right] \subset \mathbf{k}(C)[\mathbb{Z}],
$$

avec $n_{1}, \ldots, n_{s}, m_{1}, \ldots, m_{r} \in \mathbb{Z}_{>0}$, alors la présentation D.P.D. de $\bar{A}=A_{0}\left[D_{-}, D_{+}\right]$est donnée par les $\mathbb{Q}$-diviseurs

$$
D_{-}=-\min _{1 \leq i \leq r} \frac{\operatorname{div}\left(f_{i}\right)}{m_{i}} \text { et } D_{+}=-\min _{1 \leq i \leq s} \frac{\operatorname{div}\left(g_{i}\right)}{n_{i}} .
$$

Démonstration. Nous donnons la preuve pour le cas de (i). L'assertion (ii) se traite de la même façon. D'après le théorème 2.4 , on peut écrire $\bar{A}=A[C, \mathfrak{D}]$ avec $\mathfrak{D}=\sum_{z \in C} \Delta_{z} \cdot z$ où pour $z \in C$,

$$
\Delta_{z}=\left\{v \in \mathbb{Q}, m_{i} \cdot v \geq-\nu\left(f_{i}\right), 1 \leq i \leq r\right\} .
$$

Puisque $D=\mathfrak{D}(1)$, nous obtenons le résultat. 


\section{Idéaux intégralement clos et polyèdres entiers}

Dans cette section, nous rappelons une description des idéaux monomiaux intégralement clos en terme de polyèdres entiers. Nous commençons par quelques notions générales.

3.1. Puisque nous restons dans un contexte géométrique, la lettre $A$ désigne une algèbre intègre de type fini sur k. Soit $I \subset A$ un idéal. Un élément $a \in A$ est dit entier (ou satisfaisant une relation de dépendance intégrale) sur $I$ s'il existe $r \in \mathbb{Z}_{>0}$ et des éléments

$$
\lambda_{1} \in I, \lambda_{2} \in I^{2}, \ldots, \lambda_{r} \in I^{r} \text { tels que } a^{r}+\sum_{i=1}^{r} \lambda_{i} a^{r-i}=0 .
$$

L'idéal $I$ est dit intégralement clos (ou complet) si tout entier de $A$ sur $I$ appartient à $I$. On dit que $I$ est normal si pour tout entier $i \geq 1$, l'idéal $I^{i}$ est intégralement clos.

Le sous-ensemble $\bar{I} \subset A$, appelé clôture intégrale (ou fermeture intégrale) de $I$ dans $A$, est l'ensemble des éléments entiers sur $I$. C'est le plus petit idéal intégralement clos de $A$ contenant $I$ [HS, Corollary 1.3.1]. La démonstration de ce fait utilise les réductions d'idéaux (voir [NR]). Pour plus d'informations sur les idéaux intégralement clos, voir par exemple [LeTe], [HS], [Va]. Considérons l'algèbre de Rees

$$
B=A[I t]=A \oplus \bigoplus_{i \geq 1} I^{i} t^{i}
$$

correspondante à un idéal $I$ de $A$. Alors la normalisation de $B$ est

$$
\bar{B}=\bar{A} \oplus \bigoplus_{i \geq 1} \overline{\bar{A} I^{i}} t^{i}
$$

où $\bar{A}$ est la normalisation de $A$ (voir [Ri]). La normalisation de $B$ est égale à celle de $\bar{A}[\bar{A} I t]$ [HS, Proposition 5.2.4]. Si $A$ est normale alors $A[I t]$ est normale si et seulement si $I$ est normal.

Supposons maintenant que $A$ est $M$-graduée normale. Un idéal $I$ de $A$ est dit homogène si $I$ est non nul et si $I$ est engendré par des éléments homogènes de $A$. Chaque idéal homogène de $A$ est un sous- $\mathbb{T}$-module rationnel [Br, §1.1] et admet donc une décomposition en somme directe de sous-espaces propres. D'après [HS, Corollary 5.2.3], si $I$ est homogène alors $\bar{I}$ est homogène.

En l'absence de référence, nous donnons la preuve du lemme suivant.

Lemme 3.2. Soit $A$ une algèbre normale de type fini sur $\mathbf{k}$ et soit I un idéal de A. Alors la fermeture intégrale de $A[I t]$ dans son corps des fractions est égale à celle de $A[\bar{I} t]$. En particulier, pour tout $i \in \mathbb{Z}_{>0}$, $\overline{I^{i}}=\overline{I^{i}}$.

Démonstration. Pour tout $i \in \mathbb{Z}_{>0}$, on a $I^{i} \subset \bar{I}^{i}$, soit $A[I t] \subset A[\bar{I} t]$. D'où $\overline{A[I t]} \subset \overline{A[\bar{I} t]}$. Par (6) et puisque $A=\bar{A}$ est normale, $\bar{I} t$ est inclus dans $\overline{A[I t]}$, donc $A[\bar{I} t] \subset \overline{A[I t]}$ et finalement $\overline{A[\bar{I} t]} \subset \overline{A[I t]}$. La deuxième affirmation est une conséquence de (6) et de l'égalité $\overline{A[I t]}=\overline{A[\bar{I} t]}$.

3.3. Fixons un cône polyédral saillant $\sigma \subset N_{\mathbb{Q}}$. Considérons une partie $F \subset M_{\mathbb{Q}}$ telle que $F \cap \sigma^{\vee} \neq \emptyset$. On note $\operatorname{Pol}_{\sigma^{\vee}}(F)$ l'ensemble des polyèdres de la forme $P=Q+\sigma^{\vee}$ avec $Q$ un polytope dont les sommets appartiennent à $F$. Un $\sigma^{\vee}$-polyédre entier est un élément de $\operatorname{Pol}_{\sigma} \vee(M)$. 
Si $P \in \operatorname{Pol}_{\sigma^{\vee}}(M)$ alors pour tout entier $e \geq 1$, on pose $e P:=P+\ldots+P$ la somme de Minkowski de $e$ exemplaires de $P$. Le polyèdre $e P$ est l'image de $P$ par l'homothétie de centre 0 et de rapport $e$. Si $e=0$ alors on pose $e P=\sigma^{\vee}$. On dit que $P$ est normal si pour tout entier $e \geq 1$,

$$
(e P) \cap M=\left\{m_{1}+\ldots+m_{e} \mid m_{1}, \ldots, m_{e} \in P \cap M\right\} .
$$

Un sous-semi-groupe $E \subset M$ est dit saturé si pour $d \in \mathbb{Z}_{>0}$ et pour $m \in M$ tels que $d m \in E$, on a $m \in E$. Cela est équivalent à ce que $E$ soit l'intersection de Cone $(E)$ et du réseau $M$.

L'assertion suivante est aisée et nous laissons la démonstration aux lecteurs.

Lemme 3.4. Pour un cône polyédral saillant $\sigma \subset N_{\mathbb{Q}}$ et un polyèdre entier $P \in \operatorname{Pol}_{\sigma} \vee(M)$, on note

$$
S=S_{P}:=\{(m, e) \in M \times \mathbb{N} \mid m \in(e P) \cap M\} .
$$

Alors $S$ est un sous-semi-groupe saturé de $M \times \mathbb{Z}$. De plus, pour tout e $\geq 1$, l'enveloppe convexe de

$$
E_{[e, P]}:=\left\{m_{1}+\ldots+m_{e} \mid m_{1}, \ldots, m_{e} \in P \cap M\right\}
$$

dans $M_{\mathbb{Q}}$ est égale à eP.

Rappelons que tout idéal homogène intégralement clos d'une variété torique affine est caractérisé par l'enveloppe convexe de l'ensemble de ses poids. Voir [Vit, 3.1] pour le cas de l'algèbre des polynômes à plusieurs variables. Le résultat suivant est connu (voir [CLS, Proposition 11.3.4] pour le cas où $\sigma^{\vee}$ est saillant, ainsi 3 que [KKMS, Chapter I, §2] pour le cas général). Par commodité, nous donnons une courte preuve. Cela nous sera utile pour la suite. Pour tout entier $e$, nous noterons $M_{e}=M \times\{e\}$.

Théorème 3.5. Soit $\sigma \subset N_{\mathbb{Q}}$ un cône polyédral saillant. Alors l'application

$$
P \mapsto I[P]=\bigoplus_{m \in P \cap M} \mathbf{k} \chi^{m}
$$

est une bijection entre $\operatorname{Pol}_{\sigma \vee}\left(\sigma_{M}^{\vee}\right)$ et l'ensemble des idéaux homogènes intégralement clos de $A:=\mathbf{k}\left[\sigma_{M}^{\vee}\right]$. Plus précisément, si $I=\left(\chi^{m_{1}}, \ldots, \chi^{m_{r}}\right)$ est un idéal homogène de $A$ alors sa fermeture $\bar{I}$ est $I[P]$ où

$$
P=\operatorname{Conv}\left(m_{1}, \ldots, m_{r}\right)+\sigma^{\vee} .
$$

Le cône des poids $\widetilde{\omega}$ de $A[I t]$ est l'unique cône vérifiant $\widetilde{\omega} \cap M_{e}=(e P) \cap M$, pour tout $e \in \mathbb{N}$. De plus, si $P \in \operatorname{Pol}_{\sigma^{\vee}}\left(\sigma_{M}^{\vee}\right)$ alors $P$ est normal si et seulement si $I[P]$ est normal.

Démonstration. La justification de la bijectivité de $I \mapsto I[P]$ provient de [KKMS, Chapter I, §2] et nous l'omettons.

Soit $I \subset A$ un idéal engendré par $\chi^{m_{1}}, \ldots, \chi^{m_{r}}$. Considérons $P$ comme dans (7). Montrons l'égalité $\bar{I}=I[P]$. Puisque $I \subset I[P]$ et comme $I[P]$ est intégralement clos, on a $\bar{I} \subset I[P]$. Soit $\chi^{m} \in I[P]$. Alors $m \in P \cap M$. Donc il existe $c_{1}, \ldots, c_{r} \in \mathbb{Q}_{\geq 0}$ de somme égale à 1 et $c \in \sigma^{\vee}$ tels que

$$
m=\sum_{i=1}^{r} c_{i} m_{i}+c .
$$

3. Nous remercions un des rappoteurs de nous avoir communiqué cette référence. 
Soit $d^{\prime} \in \mathbb{Z}_{>0}$ tel que $d^{\prime} c_{i} \in \mathbb{N}$ pour chaque $i$ et tel que $d c \in \sigma_{M}^{\vee}$. Alors $\chi^{d^{\prime} m} \in I^{d^{\prime}}$ et donc $\chi^{m} \in \bar{I}$. D'où $\bar{I}=I[P]$ et la surjectivité de $\varphi$.

Déterminons le cône des poids $\widetilde{\omega}$ de $A[I t]$. D'après le lemme 3.2, on peut supposer que $I=I[P]$. Soit $e \in \mathbb{Z}_{>0}$. Alors on a

$$
I[P]^{e}=\bigoplus_{m \in E[e, P]} \mathbf{k} \chi^{m}
$$

Notons $\overline{I[P]^{e}}=I\left[P_{e}\right]$, pour un polyèdre $P_{e} \in \operatorname{Pol}_{\sigma \vee}\left(\sigma_{M}^{\vee}\right)$. Par le lemme 3.4, on a d'une part,

$$
e P=\operatorname{Conv}\left(E_{[e, P]}\right) \subset P_{e} .
$$

D'autre part, $I[P]^{e} \subset I[e P]$ et donc $I\left[P_{e}\right] \subset I[e P]$. D'où $e P=P_{e}$. D'après l'égalité $(6)$ de 3.1 , $\widetilde{\omega}$ est le cône des poids de

$$
\overline{A[I t]}=A \oplus \bigoplus_{e \in \mathbb{Z}_{>0}} I[e P] t^{e}
$$

D'où $\widetilde{\omega} \cap M_{e}=(e P) \cap M$, pour tout entier $e \geq 0$. Les autres assertions suivent aisément.

Le théorème suivant est une conséquence d'un résultat sur la normalité des polytopes entiers [BGN, Theorem 1.1.3].

Théorème 3.6. Soit $\sigma \subset N_{\mathbb{Q}}$ un cône polyédral saillant, notons $n=\operatorname{dim} N_{\mathbb{Q}}$ et considérons $P \in \operatorname{Pol}_{\sigma} \vee(M)$. Alors pour tout entier $e \geq n-1$, le polyèdre eP est normal. En particulier, tout idéal stable intégralement clos d'une surface torique est normal.

Démonstration. Soit $\mathscr{E}$ une subdivision de cônes polyédraux saillants de $\sigma^{\vee}$ de dimension $n$. Ecrivons $P=Q+\sigma^{\vee}$ avec $Q$ un polytope entier. Soit $m \in(e P) \cap M$. Alors il existe $\tau \in \mathscr{E}$ tel que $m \in(e Q+\tau) \cap M$. D'après [CLS, Proposition 7.1.9], on a $m \in E_{[e, Q+\tau]}$ (voir 3.4). Donc $m \in E_{[e, P]}$ et $e P$ est normal. Le reste provient du théorème 3.5 .

Remarque 3.7. Notons que dans le cas où $\mathbf{k}\left[\sigma_{M}^{\vee}\right]=\mathbf{k}\left[x_{1}, \ldots, x_{n}\right]$ est l'algèbre des polynômes, un idéal monomial $I \subset \mathbf{k}\left[x_{1}, \ldots, x_{n}\right]$ est normal si et seulement si pour tout $i=1, \ldots, n-1$, l'idéal $I^{i}$ est intégralement clos [RRV, 3.1]. Nous verrons une généralisation de ce résultat dans la section 5 .

D'après [ZS, Appendix 5], [HS, §1.1.4, §14.4.4], tout idéal intégralement clos d'une surface affine lisse est normal. Cependant cela ne s'applique pas aux variétés toriques affines de dimension 3 . En effet, d'après [HS, Exercice 1.14], si $\sigma \subset \mathbb{Q}^{3}$ est l'octant positif et si

$$
P:=\operatorname{Conv}((2,0,0),(0,3,0),(0,0,7))+\sigma^{\vee}
$$

alors $I[P]$ n'est pas normal.

\section{Idéaux intégralement clos et $\mathbb{T}$-variétés affines de complexité un}

Dans cette section, $C$ désigne une courbe algébrique lisse, le sous-ensemble $\sigma \subset N_{\mathbb{Q}}$ est un cône polyédral saillant et $\mathfrak{D}=\sum_{z \in C} \Delta_{z} \cdot z$ est un diviseur $\sigma$-polyédral propre. Posons $A:=A[C, \mathfrak{D}]$. Nous allons étudier les idéaux homogènes intégralement clos de $A$. Nous commençons par décrire le cône des poids de l'algèbre de Rees de $A$ selon un idéal homogène. Rappelons que pour un entier $e, M_{e}$ désigne $M \times\{e\}$. 
Lemme 4.1. Soit $I \subset A$ un idéal engendré par des éléments homogènes $f_{1} \chi^{m_{1}}, \ldots, f_{r} \chi^{m_{r}}$. Notons

$$
P=\operatorname{Conv}\left(m_{1}, \ldots m_{r}\right)+\sigma^{\vee} .
$$

Alors le cône des poids $\widetilde{\omega}$ de l'algèbre $(M \times \mathbb{Z})$-graduée $A[I t]$ vérifie $\widetilde{\omega} \cap M_{e}=(e P) \cap M$, pour tout $e \in \mathbb{N}$. Démonstration. Soit $E$ l'ensemble des poids de $I$. La partie $J:=\bigoplus_{m \in E} \mathbf{k} \chi^{m}$ est un idéal de $A^{\prime}:=\mathbf{k}[S]$. Par le lemme 3.2 , on a

$$
\overline{A^{\prime}[J t]}=\overline{B[\overline{J B} t]} \text { avec } B:=\mathbf{k}\left[\sigma_{M}^{\vee}\right] \text {. }
$$

Les éléments $\chi^{m_{1}}, \ldots, \chi^{m_{r}}$ engendrent l'idéal $J B$. Puisque $\widetilde{\omega}$ est le cône des poids de $\overline{A^{\prime}[J t]}$, le reste provient du théorème 3.5 .

L'assertion suivante permet de calculer explicitement la fermeture intégrale des idéaux homogènes de l'algèbre $A$.

Théorème 4.2. Soit I un idéal de $A=A[C, \mathfrak{D}]$ engendré par des éléments homogènes $f_{1} \chi^{m_{1}}, \ldots, f_{r} \chi^{m_{r}}$. Alors on a

$$
A=\bigoplus_{m \in \sigma_{M}^{\vee}} H^{0}\left(C, \mathcal{O}_{C}(\lfloor\widetilde{\mathfrak{D}}(m, 0)\rfloor)\right) \chi^{m}
$$

et pour tout entier $e \geq 1$,

$$
\overline{I^{e}}=\bigoplus_{m \in(e P) \cap M} H^{0}\left(C, \mathcal{O}_{C}(\lfloor\widetilde{\mathfrak{D}}(m, e)\rfloor)\right) \chi^{m}
$$

où $(P, \widetilde{\mathfrak{D}})$ vérifie les conditions suivantes.

(i) $P$ est le polyèdre $\operatorname{Conv}\left(m_{1}, \ldots, m_{r}\right)+\sigma^{\vee}$;

(ii) $\widetilde{\mathfrak{D}}$ est le diviseur polyédral sur $C$ dont ses coefficients sont définis par

$$
\widetilde{\Delta}_{z}=\bigcap_{i=1}^{r}\left\{(v, p) \in N_{\mathbb{Q}} \times \mathbb{Q}, m_{i}(v)+p \geq-\nu_{z}\left(f_{i}\right)\right\} \cap\left(\Delta_{z} \times \mathbb{Q}\right),
$$

pour tout $z \in C$.

Démonstration. On applique le théorème 2.4 à l'algèbre $A[I t]$ en utilisant les éléments $f_{i} \chi^{m_{i}}$ et des générateurs homogènes de $A$. Le diviseur polyédral correspondant $\widetilde{\mathfrak{D}}$ vérifie (ii). On conclut par le lemme 4.1 .

Exemple 4.3. Reprenons l'exemple 2.5. On considère l'idéal homogène

$$
I=\left(t_{2}, t_{3}, t_{4}\right) \subset A=k\left[t_{1}, t_{2}, t_{3}, t_{4}\right] \cong \frac{k\left[x_{1}, x_{2}, x_{3}, x_{4}\right]}{\left(x_{4}^{2}-x_{1}^{2} x_{2}^{2} x_{3}-x_{1} x_{3}^{2}\right)} .
$$

Soit $\widetilde{\omega} \subset \mathbb{Q}^{3}$ le cône vérifiant $\widetilde{\omega} \cap \mathbb{Z}_{e}^{2}=(0, e)+\mathbb{Q}_{\geq 0}^{2}$, pour tout entier $e \geq 0$. Soit $\widetilde{\mathfrak{D}}$ le diviseur $\widetilde{\omega}^{\vee}$-polyédral sur $\mathbb{P}^{1}$ construit par les générateurs $t_{2}, t_{3}, t_{4}$. Les coefficients non triviaux de $\widetilde{\mathfrak{D}}$ sont

$$
\widetilde{\Delta}_{0}=-\left(\frac{1}{2}, 0,0\right)+\widetilde{\omega}^{\vee}, \widetilde{\Delta}_{1}=\left(\frac{1}{2}, 0,0\right)+\widetilde{\omega}^{\vee}, \widetilde{\Delta}_{\infty}=\operatorname{Conv}\left((0,1,-1),\left(\frac{1}{2}, 0,0\right),\left(0, \frac{1}{2}, 0\right)\right)+\widetilde{\omega}^{\vee} .
$$


Fixons un polyèdre $P \in \operatorname{Pol}_{\sigma^{\vee}}\left(\sigma_{M}^{\vee}\right)$. Considérons un cône $\widetilde{\omega} \subset M_{\mathbb{Q}} \times \mathbb{Q}$ satisfaisant la relation

$$
\widetilde{\omega} \cap M_{e}=(e P) \cap M,
$$

pour tout $e \in \mathbb{N}$. Dans les deux prochains lemmes, nous étudions des diviseurs $\widetilde{\omega}^{\vee}$-polyédraux dont les pièces graduées correspondantes à $M_{e}$ forment un idéal de $A$.

Lemme 4.4. Soit $\widetilde{\mathfrak{D}}=\sum_{z \in C} \widetilde{\Delta}_{z} \cdot z$ un diviseur $\widetilde{\omega}^{\vee}$-polyédral propre. Alors les conditions suivantes sont équivalentes.

(i) Pour tout $z \in C$, le polyèdre $\Delta_{z}$ est la projection orthogonale de $\widetilde{\Delta}_{z}$ parallèlement à $\mathbb{Q}\left(0_{M}, 1\right)$ et si $(v, p)$ est un sommet de $\widetilde{\Delta}_{z}$ alors $p \leq 0$;

(ii) Pour tout entier $e \geq 0$,

$$
I_{[e]}:=\bigoplus_{m \in(e P) \cap M} H^{0}\left(C, \mathcal{O}_{C}(\lfloor\widetilde{\mathfrak{D}}(m, e)\rfloor)\right) \chi^{m}
$$

est un idéal de $A$ et $I_{[0]}=A$.

Démonstration. Soit $z \in C$. Notons $\Delta_{z}^{\prime}$ la projection orthogonale de $\widetilde{\Delta}_{z}$ parallèlement à l'axe $\mathbb{Q}\left(0_{M}, 1\right)$. Montrons l'implication (i) $\Rightarrow$ (ii). Puisque pour $m \in \sigma^{\vee}, h_{\widetilde{\Delta}_{z}}(m, 0)=h_{\Delta_{z}^{\prime}}(m)$, on a l'égalité $I_{[0]}=A$. Le lemme 1.10 implique que pour tout $e \in \mathbb{N}, I_{[e]} \subset A$. D'où l'assertion (ii).

Réciproquement, la propreté de $\mathfrak{D}$ et l'égalité $A=I_{[0]}$ montre que $\Delta_{z}=\Delta_{z}^{\prime}$. Soit $(v, p)$ un sommet de $\widetilde{\Delta}_{z}$. Il reste à montrer que $p \leq 0$. La partie

$$
\lambda:=\left\{(m, e) \in M_{\mathbb{Q}} \times \mathbb{Q}, h_{\widetilde{\Delta}_{z}}(m, e)=m(v)+e p\right\}
$$

est un cône d'interieur non vide $[\mathrm{AH}, \S 1]$. Donc l'ensemble $\lambda_{M \times \mathbb{Z}}=\lambda \cap(M \times \mathbb{Z})$ contient un élément $\left(m^{\prime}, e^{\prime}\right)$ vérifiant $e^{\prime} \geq 1$. Soit $v^{\prime} \in \Delta_{z}$ tels que

$$
m^{\prime}\left(v^{\prime}\right)=h_{\Delta_{z}}\left(m^{\prime}\right)
$$

Par propreté de $\widetilde{\mathfrak{D}}$ et [AH, Lemma 9.1], il s'ensuit que

$$
m^{\prime}(v)+e^{\prime} p=h_{\widetilde{\Delta}_{z}}\left(m^{\prime}, e^{\prime}\right) \leq h_{\Delta_{z}}\left(m^{\prime}\right)=m^{\prime}\left(v^{\prime}\right) .
$$

Comme $v$ appartient à $\Delta_{z}$, on a $m^{\prime}\left(v^{\prime}\right) \leq m^{\prime}(v)$ et donc $p \leq 0$. D'où le résultat.

Lemme 4.5. Soit $\widetilde{\mathfrak{D}}$ un diviseur $\widetilde{\omega}^{\vee}$-polyédral sur $C$. Supposons que $\widetilde{\mathfrak{D}}$ vérifie la condition (ii) du lemme 4.4. Alors pour tout $e \in \mathbb{N}, I_{[e]}$ est un idéal homogène intégralement clos.

Démonstration. Soit $e \in \mathbb{N}$. Il suffit de montrer que tout élément homogène de $\bar{I}_{[e]}$ appartient à $I_{[e]}$. Soit $a \in \bar{I}_{[e]}$ un élément homogène. L'élément $a \chi^{(0, e)}$ appartient à la normalisation de $A[C, \widetilde{\mathfrak{D}}]$. Puisque $A[C, \widetilde{\mathfrak{D}}]$ est normal [De, §2.7], nous avons $a \in I_{[e]}$.

Le théorème suivant décrit les idéaux homogènes intégralement clos pour la compléxité 1 . Soit $S$ le semi-groupe des poids de $A$. Dans ce théorème, nous considérons des couples $(P, \widetilde{\mathfrak{D}})$ vérifiant les conditions suivantes.

(i) $P$ est un polyèdre de $\operatorname{Pol}_{\sigma} \vee(S)$; 
(ii) $\widetilde{\mathfrak{D}}=\sum_{z \in C} \widetilde{\Delta}_{z} \cdot z$ est un diviseur $\widetilde{\omega}^{\vee}$-polyédral où $\widetilde{\omega} \subset M_{\mathbb{Q}} \times \mathbb{Q}$ vérifie

$$
\widetilde{\omega} \cap M_{e}=(e P) \cap M,
$$

pour tout entier $e \geq 0$;

(iii) Pour tout $z \in C, \Delta_{z}$ est la projection orthogonale de $\widetilde{\Delta}_{z}$ parallèlement à $\mathbb{Q}\left(0_{M}, 1\right)$ et tout sommet $(v, p) \in \widetilde{\Delta}_{z}$ satisfait $p \leq 0$

(iv) Pour tout $z \in C, \widetilde{\Delta}_{z}$ est l'intersection dans $\Delta_{z} \times \mathbb{Q}$ d'un nombre fini de demi-espaces

$$
\Delta_{m, e}:=\left\{(v, p) \in N_{\mathbb{Q}} \times \mathbb{Q}, m(v)+p \geq e\right\}
$$

avec $e \in \mathbb{Z}$ et $m \in P \cap M$ tel que les sections globales du faisceau $\mathcal{O}_{C}(\lfloor\widetilde{\mathfrak{D}}(m, 1)\rfloor)$ engendrent le germe $\mathcal{O}_{C}(\lfloor\widetilde{\mathfrak{D}}(m, 1)\rfloor)_{z}$.

Théorème 4.6. Soit $\mathfrak{D}=\sum_{z \in C} \Delta_{z} \cdot z$ un diviseur $\sigma$-polyédral propre et soit $A=A[C, \mathfrak{D}]$. Alors il existe une bijection entre l'ensemble des idéaux homogènes intégralement clos de $A$ et l'ensemble des couples $(P, \widetilde{\mathfrak{D}})$ vérifiant $(\mathrm{i})-(\mathrm{iv})$. Cette correspondance est donnée par

$$
(P, \widetilde{\mathfrak{D}}) \mapsto I=\bigoplus_{m \in P \cap M} H^{0}\left(C, \mathcal{O}_{C}(\lfloor\widetilde{\mathfrak{D}}(m, 1)\rfloor)\right) \chi^{m} .
$$

De plus, sous cette correspondance, l'algèbre $A[C, \widetilde{\mathfrak{D}}]$ s'identifie à la normalisation de l'algèbre de Rees $A[I t]$.

Démonstration. Soit $(P, \widetilde{\mathfrak{D}})$ un couple vérifiant les conditions (i) - (iv). Montrons que $\widetilde{\mathfrak{D}}$ est propre. Soient $z_{1}, \ldots, z_{s}$ les points du support de $\widetilde{\mathfrak{D}}$. D'après (iv), on a pour $j=1, \ldots, s$,

$$
\widetilde{\Delta}_{z_{j}}=\left(\Delta_{z} \times \mathbb{Q}\right) \cap \bigcap_{i=1}^{r_{j}} \Delta_{m_{i j}, e_{i j}}
$$

avec $e_{i j} \in \mathbb{Z}$ et $m_{i j} \in P \cap M$ tel qu'il existe $f_{i j} \chi^{\left(m_{i j}, 1\right)} \in A[C, \tilde{\mathfrak{D}}]$ homogène satisfaisant $\nu_{z_{j}}\left(f_{i j}\right)=$ $h_{\widetilde{\Delta}_{z_{j}}}\left(m_{i j}, 1\right)$. Considérons l'algèbre $B$ engendrée par $A$ et par les éléments

$$
f_{i j} \chi^{\left(m_{i j}, 1\right)}, 1 \leq j \leq s, 1 \leq i \leq r_{j}
$$

Par le théorème 4.2 , l'anneau $A[C, \widetilde{\mathfrak{D}}]$ est la normalisation de $B$. Ce qui donne la propreté de $\widetilde{\mathfrak{D}}$.

Les lemmes 4.4 et 4.5 montrent que l'application (8) est bien définie. Ainsi, $A[C, \widetilde{\mathfrak{D}}]=\overline{A[I t]}$ où $t=\chi^{(0,1)}$. Par l'égalité (6) de 3.1, on déduit l'injectivité.

Montrons la surjectivité. Soit $I$ un idéal intégralement clos de $A$ engendré par des éléments homogènes $f_{1} \chi^{m_{1}}, \ldots, f_{r} \chi^{m_{r}}$. Considérons le couple $(P, \widetilde{\mathfrak{D}})$ obtenu à partir du théorème 4.2. Des lemmes $4.1,4.4$ et 4.5, on obtient (i), (ii), (iii). Montrons (iv). Pour un point $z \in C$, écrivons

$$
\widetilde{\Delta}_{z}=\left(\Delta_{z} \times \mathbb{Q}\right) \cap \bigcap_{i=1}^{r} \Delta_{m_{i}, e_{i}}
$$

avec pour tout $i, e_{i}:=-\nu_{z}\left(f_{i}\right)$. Soit $E$ un sous-ensemble minimal de $\{1, \ldots, r\}$ tel que

$$
\widetilde{\Delta}_{z}=\left(\Delta_{z} \times \mathbb{Q}\right) \cap \bigcap_{i \in E} \Delta_{m_{i}, e_{i}}
$$


Fixons un élément $i^{\prime} \in E$. Alors on a

$$
\left\{(v, p) \in N_{\mathbb{Q}} \times \mathbb{Q}, m_{i^{\prime}}(v)+p=e_{i^{\prime}}\right\} \cap \widetilde{\Delta}_{z} \neq \emptyset .
$$

Donc $h_{\widetilde{\Delta}_{z}}\left(m_{i^{\prime}}, 1\right)=e_{i^{\prime}}=-\nu_{z}\left(f_{i^{\prime}}\right)$. D'où la condition (iv).

Lorsque $C$ est affine, tout fibré en droite au-dessus de $C$ est globalement engendré. Ainsi, comme conséquence immédiate, on a le résultat suivant.

Corollaire 4.7. Supposons que $C$ est affine. Alors il existe une bijection entre l'ensemble des idéaux homogènes intégralement clos de $A$ et l'ensemble des couples $(P, \widetilde{\mathfrak{D}})$ vérifiant (ii), (iii) du théorème 4.6 et les conditions suivantes.

(i)' $P$ est un polyèdre de $\operatorname{Pol}_{\sigma^{\vee}}\left(\sigma_{M}^{\vee}\right)$;

(iv)' Pour tout $z \in C$, le polyèdre $\widetilde{\Delta}_{z}$ est l'intersection dans $\Delta_{z} \times \mathbb{Q}$ d'un nombre fini de demi-espaces

$$
\Delta_{m, e}:=\left\{(v, p) \in N_{\mathbb{Q}} \times \mathbb{Q}, m(v)+p \geq e\right\}
$$

avec $e \in \mathbb{Z}$ et $m \in P \cap M$.

La correspondance est donnée par l'application (8) de 4.6.

Remarque 4.8. Soient $g_{2}, g_{3} \in \mathbf{k}$ tels que $g_{2}^{3}-27 g_{3}^{2} \neq 0$. Considérons la courbe elliptique $C \subset \mathbb{P}^{2}$ d'équation de Weierstrass $y^{2}=4 x^{3}-g_{2} x-g_{3}, O$ son point à l'infini et $A$ l'algèbre graduée

$$
\bigoplus_{m \in \mathbb{N}} H^{0}\left(C, \mathcal{O}_{C}(m \cdot O)\right) \chi^{m}
$$

Soit $\widetilde{\omega} \subset \mathbb{Q}^{2}$ le cône

$$
\mathbb{Q}_{\geq 0}(1,0)+\mathbb{Q}_{\geq 0}(1,1),
$$

$P=\mathbb{Q}_{\geq 1}$ et $\widetilde{\mathfrak{D}}:=\widetilde{\Delta} \cdot O$ le diviseur polyédral sur $C$ avec $\widetilde{\Delta}=(1,0)+\widetilde{\omega}^{\vee}$. Alors $(P, \widetilde{\mathfrak{D}})$ vérifie les conditions (i), (ii), (iii) et (iv)' de 4.6 et 4.7 . Cependant la condition (iv) n'est pas satisfaite. L'algèbre $A[C, \widetilde{\mathfrak{D}}]$ est donc distincte de la normalisation de $A[I t]$ où $I$ est l'idéal provenant de $(8)$ et $t:=\chi^{(0,1)}$.

\section{Exemples d'idéaux homogènes normaux}

Dans cette section, nous considérons l'algèbre $A=A[C, \mathfrak{D}]$ comme dans la section 4 . Supposons que $C$ est affine et fixons un idéal homogène intégralement $\operatorname{clos} I \subset A$. Nous allons donner des conditions sur le couple $(P, \widetilde{\mathfrak{D}})$ associé (voir 4.6$)$ pour que $I$ soit normal. Comme d'habitude, nous notons $\widetilde{\Delta}_{z}$ le coefficient de $\widetilde{\mathfrak{D}}$ au point $z \in C$.

Notation 5.1. Pour tout $z \in C$, nous considérons le sous-ensemble

$$
\widetilde{P}_{z}:=\operatorname{Conv}\left(\left\{(m, i) \in(P \cap M) \times \mathbb{Z}, h_{\widetilde{\Delta}_{z}}(m, 1) \geq-i\right\}\right) .
$$

On vérifie aisément que $\widetilde{P}_{z}$ est un polyèdre entier de $M_{\mathbb{Q}} \times \mathbb{Q}$. Par ailleurs, pour un ouvert non vide $U \subset C$, $\widetilde{\mathfrak{D}} \mid U:=\sum_{z \in U} \widetilde{\Delta}_{z} \cdot z$ est le diviseur polyédral sur $U$ obtenu par restriction de $\widetilde{\mathfrak{D}}$. 
L'assertion suivante est inspirée de la description de $X[C, \widetilde{\mathfrak{D}}]$ comme variété toroïdale (voir [LS,§2.6], [KKMS, Chapter 2, 4]).

Lemme 5.2. Soit $z \in C$. Supposons que $C$ est affine et que $\mathfrak{D}, \widetilde{\mathfrak{D}}$ ont au plus le point $z$ dans leurs supports. Si le polyèdre $\widetilde{P}_{z}$ est normal alors l'idéal I est normal.

Démonstration. Fixons $e \in \mathbb{Z}_{>0}$. Déterminons un sous-ensemble générateur de $\overline{I^{e}}$. Pour tout vecteur $(m, i) \in M \times \mathbb{Z}$, posons

$$
B_{(m, i)}:=H^{0}\left(C, \mathcal{O}_{C}(-i \cdot z)\right) \chi^{m}
$$

Si $m \in(e P) \cap M$ alors on a l'égalité

$$
H^{0}\left(C, \mathcal{O}_{C}(\lfloor\widetilde{\mathfrak{D}}(m, e)\rfloor)\right) \chi^{m}=\bigcup_{i \in \mathbb{Z}, i \geq-h_{z, e}(m)} B_{(m, i)}
$$

où $h_{z, e}(m):=h_{\widetilde{\Delta}_{z}}(m, e)$. Donc l'idéal $\overline{I^{e}}$ est engendré par

$$
\bigcup_{(m, i) \in\left(e \widetilde{P}_{z}\right) \cap(M \times \mathbb{Z})} B_{(m, i)} .
$$

Montrons que pour tout $(m, i) \in\left(e \widetilde{P}_{z}\right) \cap(M \times \mathbb{Z})$, la partie $B_{(m, i)}$ est incluse dans $I^{e}$. Fixons un tel couple $(m, i)$. Par normalité de $\widetilde{P}_{z}$, il existe

$$
\left(m_{1}, i_{1}\right), \ldots,\left(m_{e}, i_{e}\right) \in \widetilde{P}_{z} \cap(M \times \mathbb{Z}) \text { tels que } \sum_{j=1}^{e}\left(m_{j}, i_{j}\right)=(m, i) .
$$

Pour chaque $j=1, \ldots, e, B_{\left(m_{j}, i_{j}\right)}$ est contenu dans $I$. Puisque la multiplication

$$
B_{\left(m_{1}, i_{1}\right)} \otimes \ldots \otimes B_{\left(m_{e}, i_{e}\right)} \rightarrow B_{(m, i)}
$$

est surjective, on a $B_{(m, i)} \subset I^{e}$. D'où le résultat.

Le théorème suivant se déduit du lemme précédent par localisation. Il peut être vu comme un analogue de l'assertion 3.6.

Théorème 5.3. Supposons que $C$ est affine. Soit $I \subset A=A[C, \mathfrak{D}]$ un idéal homogène intégralement clos et $(P, \widetilde{\mathfrak{D}})$ le couple correspondant. Si pour tout point $z$ appartenant au support de $\widetilde{\mathfrak{D}}$, le polyèdre $\widetilde{P}_{z}$ est normal alors l'idéal I est normal. Pour tout entier $e \geq n:=\operatorname{dim} N_{\mathbb{Q}}$, l'idéal $\overline{I^{e}}$ est normal. En particulier, tout idéal stable intégralement clos d'une $\mathbf{k}^{\star}$-surface affine non elliptique est normal.

Démonstration. Notons $z_{1}, \ldots, z_{r}$ les points distincts du support de $\widetilde{\mathfrak{D}}$. Par le théorème des restes chinois, l'application

$$
\pi: \mathbf{k}[C] \rightarrow \mathbf{k}^{r}, \quad f \mapsto\left(f\left(z_{1}\right), \ldots, f\left(z_{r}\right)\right)
$$

est surjective. Soit $\left(e_{1}, \ldots, e_{r}\right)$ la base canonique de $\mathbf{k}^{r}$ et pour $i=1, \ldots, r$, prenons $f_{i}$ un élément de $\pi^{-1}\left(\left\{e_{i}\right\}\right)$. Pour une fonction régulière $f \in \mathbf{k}[C]$, nous notons $C_{f}=C-V(f)$ son lieu de non annulation. Soient $f_{r+1}, f_{r+2}, \ldots, f_{s} \in \mathbf{k}[C]$ tels que

$$
U:=C-\left\{z_{1}, \ldots, z_{r}\right\}=\bigcup_{i=r+1}^{s} C_{f_{j}}
$$


Alors on a l'égalité

$$
C=\bigcup_{i=1}^{s} C_{f_{i}} .
$$

Considérons $z_{r+1}, z_{r+2}, \ldots, z_{s} \in U$ des points tels que $f_{i}\left(z_{i}\right) \neq 0$ pour $i=r+1, \ldots, s$. Fixons un entier $i \in \mathbb{N}$ tel que $1 \leq i \leq s$. Puisque $\widetilde{P}_{z_{i}}$ est un polyèdre entier normal, par le lemme 5.2, l'idéal

$$
I_{f_{i}}:=\bigoplus_{m \in P \cap M} H^{0}\left(C_{f_{i}}, \mathcal{O}_{C}(\lfloor\widetilde{\mathfrak{D}}(m, 1)\rfloor)\right) \chi^{m}=I \otimes_{\mathbf{k}[C]} \mathbf{k}\left[C_{f_{i}}\right] \subset A \otimes_{\mathbf{k}[C]} \mathbf{k}\left[C_{f_{i}}\right]
$$

correspondant au couple $\left(P, \widetilde{\mathfrak{D}} \mid C_{f_{i}}\right)$ est normal. Donc nous avons

$$
A\left[C_{f_{i}}, \widetilde{\mathfrak{D}} \mid C_{f_{i}}\right]=\overline{\left(A \otimes_{\mathbf{k}[C]} \mathbf{k}\left[C_{f_{i}}\right]\right)\left[I_{f_{i}} t\right]}=\left(A \otimes_{\mathbf{k}[C]} \mathbf{k}\left[C_{f_{i}}\right]\right)\left[I_{f_{i}} t\right]=A[I t] \otimes_{\mathbf{k}[C]} \mathbf{k}\left[C_{f_{i}}\right] .
$$

Écrivons

$$
A[I t]=\bigoplus_{(m, e) \in \widetilde{\sigma}_{M \times \mathbb{Z}}^{\vee}} I_{(m, e)} \chi^{m} t^{e}
$$

avec $I_{(m, e)} \subset \mathbf{k}(C)$, pour tout $(m, e)$. Alors par (11), le semi-groupe des poids de $A[I t]$ est $\tilde{\sigma}_{M \times \mathbb{Z} \cdot \text { Cela }}^{\vee}$ implique que chaque $I_{(m, e)}$ est un idéal fractionnaire de $\mathbf{k}[C]$. Donc pour tout vecteur $(m, e) \in \widetilde{\sigma}_{M \times \mathbb{Z}}^{\vee}$, il existe un diviseur de Weil entier $D_{(m, e)}$ sur $C$ tel que

$$
I_{(m, e)}=H^{0}\left(C, \mathcal{O}_{C}\left(D_{(m, e)}\right)\right) .
$$

Comme

$$
A[I t] \otimes_{\mathbf{k}[C]} \mathbf{k}\left[C_{f_{i}}\right]=\bigoplus_{(m, e) \in \widetilde{\sigma}_{M \times \mathbb{Z}}^{\vee}} H^{0}\left(C_{f_{i}}, \mathcal{O}_{C}\left(D_{(m, e)}\right)\right) \chi^{m} t^{e}
$$

on a d'une part,

$$
\bigcap_{i=1}^{s} A[I t] \otimes_{\mathbf{k}[C]} \mathbf{k}\left[C_{f_{i}}\right]=A[I t]
$$

et d'autre part,

$$
\bigcap_{i=1}^{s} A\left[C_{f_{i}}, \widetilde{\mathfrak{D}} \mid C_{f_{i}}\right]=A[C, \widetilde{\mathfrak{D}}],
$$

on conclut par (11) que $A[I t]=A[C, \widetilde{\mathfrak{D}}]$. Cela donne la normalité de $I$. Le reste de la preuve est une conséquence directe du théorème 3.6.

La prochaine assertion est une traduction combinatoire de [RRV, Proposition 3.1] via la correspondance du théorème 3.5 .

Lemme 5.4. Soit $n \in \mathbb{N}$ un entier, notons $\sigma^{\vee}=\mathbb{Q}_{\geq 0}^{n+1}$ et soit $P$ un $\sigma^{\vee}$-polyèdre entier contenu dans $\sigma^{\vee}$. Alors les assertions suivantes sont équivalentes.

(i) Le polyèdre est normal;

(ii) Pour tout $s \in\{1, \ldots, n\}$, on a l'égalité $(s P) \cap \mathbb{Z}^{n+1}=E_{[s, P]}$ (voir 3.2). 
Comme application du théorème 5.3, nous obtenons une caractérisation de la normalité pour une classe d'idéaux de l'algèbre des polynômes.

Corollaire 5.5. Soit $n \in \mathbb{Z}_{>0}$. Considérons l'algèbre des polynômes $\mathbf{k}^{[n+1]}=\mathbf{k}\left[x_{0}, x_{1}, \ldots, x_{n}\right]$ à $n+1$ variables munie de la $\mathbb{Z}^{n}$-graduation

$$
\mathbf{k}^{[n+1]}=\bigoplus_{\left(m_{1}, \ldots, m_{r}\right) \in \mathbb{N}^{n}} \mathbf{k}\left[x_{0}\right] x_{1}^{m_{1}} \ldots x_{n}^{m_{n}}
$$

et soit I un idéal homogène de A. Alors les assertions suivantes sont équivalentes.

(i) L'idéal I est normal;

(ii) Pour tout $e \in\{1, \ldots, n\}$, l'idéal $I^{e}$ est intégralement clos.

Démonstration. Posons $C:=\mathbb{A}^{1}=\operatorname{Spec} \mathbf{k}\left[x_{0}\right], \sigma:=\mathbb{Q}_{\geq 0}^{n}$ et $M:=\mathbb{Z}^{n}$. Considérons le diviseur $\sigma$-polyédral $\mathfrak{D}$ sur la courbe $C$ dont l'évaluation est identiquement nulle. Alors on a $\mathbf{k}^{[n+1]}=A=A[C, \mathfrak{D}]$.

Montrons l'implication (ii) $\Rightarrow\left(\right.$ i). Soit $(P, \widetilde{D})$ le couple correspondant à l'idéal $I$. Notons $z_{1}, \ldots, z_{r}$ les points distincts du support de $\widetilde{\mathfrak{D}}$. Pour $i=1, \ldots, r$, considérons le polynôme

$$
f_{i}\left(x_{0}\right)=\prod_{j \neq i}\left(x_{0}-z_{j}\right)
$$

Alors pour tout $i$, nous avons

$$
I_{f_{i}}:=I \otimes_{\mathbf{k}[C]} \mathbf{k}\left[C_{f_{i}}\right]=\underset{(m, e) \in \widetilde{P}_{z_{i}} \cap(M \times \mathbb{Z})}{\bigoplus} \mathbf{k}\left[\frac{1}{f_{i}}\right]\left(x_{0}-z_{i}\right)^{e} \chi^{m}
$$

où pour $m=\left(m_{1}, \ldots, m_{r}\right), \chi^{m}:=x_{1}^{m_{1}} \ldots x_{n}^{m_{n}}$. Fixons $s \in\{1, \ldots, n\}$. Alors on a d'une part,

$$
I_{f_{i}}^{s}=\bigoplus_{(m, e) \in E_{\left[s, \widetilde{P}_{\left.z_{i}\right]}\right.}} \mathbf{k}\left[\frac{1}{f_{i}}\right]\left(x_{0}-z_{i}\right)^{e} \chi^{m}
$$

et d'autre part,

$$
\overline{I_{f_{i}}^{s}}=\bigoplus_{m \in(s P) \cap M} H^{0}\left(C_{f_{i}}, \mathcal{O}(\lfloor\widetilde{\mathfrak{D}}(m, e)\rfloor)\right) \chi^{m}=\underset{(m, e) \in\left(s \widetilde{P}_{z_{i}}\right) \cap(M \times \mathbb{Z})}{\bigoplus} \mathbf{k}\left[\frac{1}{f_{i}}\right]\left(x_{0}-z_{i}\right)^{e} \chi^{m},
$$

comparer avec [HS, Proposition 1.1.4]. Puisque $I^{s}$ est intégralement clos, l'idéal $I_{f_{i}}^{s} \subset A_{f_{i}}$ l'est encore. Ce qui donne par les égalités précédentes,

$$
\left(s \widetilde{P}_{z_{i}}\right) \cap(M \times \mathbb{Z})=E_{\left[s, \widetilde{P}_{z_{i}}\right]} .
$$

En appliquant le lemme 5.4, on déduit que $\widetilde{P}_{z_{i}}$ est normal. Par le théorème 5.3, on obtient que $I$ est normal. D'où l'implication (ii) $\Rightarrow$ (i). La réciproque est aisée. 


\section{Références}

[AH] K. Altmann, J. Hausen. Polyhedral divisors and algebraic torus actions. Math. Ann. 334. (2006). 557-607.

[AHS] K. Altmann, J. Hausen, H. Süss. Gluing affine torus actions via divisorial fans. Transform. Groups. 13. (2008). 215-242.

[AOPSV] K. Altmann, N. Owen Ilten, L. Petersen, H. Süss, R. Volmert. The geometry of T-varieties. IMPANGA Lecture Notes. Contributions to Algebraic Geometry. (2012). 17-69.

[AFKKZ] I. Arzhantsev, H. Flenner, S. Kaliman, F. Kutzschebauch, M. Zaidenberg. Flexible varieties and automorphism groups. Duke Math. J. 162. (2013). 767-823.

[AKZ] I.V. Arzhantsev, K. Kuyumzhiyan, M. Zaidenberg. Flag varieties, toric varieties, and suspensions : three instances of infinite transitivity. Mat. Sbornik. 203. (2012). 3-30.; English translation : Sbornik Math. 203. (2012). 923-949.

[AL] I.V. Arzhantsev, A. Liendo. Polyhedral divisors and $S L_{2}$-actions on affine T-varieties. Michigan Math. J. 61. (2012). 731-762.

[Br] M. Brion. Sur la géométrie des variétés sphériques. Comment. Math. Helv. 66. (1991). 237-262.

[BGN] W. Bruns, J. Gubeladze, T. Ngô Viêt. Normal polytopes, triangulations, and Koszul algebras. J. Reine Angew. Math. 485. (1997). 123-160.

[CLS] D. Cox, J. Little, H. Schenck. Toric Varieties. Graduate Studies in Mathematics. AMS. 124. (2011).

[Da] V.I. Danilov. The Geometry of Toric Varieties. Russian Math. Surveys. 33. (1978). 97-154.

[De] M. Demazure. Anneaux gradués normaux. Trav. Cours 37. (1988). 35-68.

[Du] A. Dubouloz. Quelques remarques sur la notion de modification affine. arxiv : 0503142. (2005). 5p.

[FZ] H. Flenner, M. Zaidenberg. Normal affine surfaces with $\mathbb{C}^{\star}$-actions. Osaka J.Math. 40. (2003). 981-1009.

[Fu] W. Fulton. Introduction to toric varieties. Princeton University Press. 131. (1993).

[Ha] R. Hartshorne. Algebraic Geometry. Graduate Texts in Mathematics. Springer-Verlag. 52. (1977).

[Ho] M. Hochster. Rings of invariants of tori, Cohen-Macaulay rings generated by monomials and Polytopes. Ann. of Math. 96. (1972). 318-337.

[HS] C. Huneke, I. Swanson. Integral Closure of Ideals, Rings and Modules. Polycopié : http ://people.reed.edu/ iswanson/book/.

[KZ] S. Kaliman, M. Zaidenberg. Affine modifications and affine hypersurfaces with a very transitive automorphism group. Transform. Groups. 4. (1999). 53-95.

[KKMS] G. Kempf, F. Knudsen, D. Mumford, and B. Saint-Donat. Toroidal embeddings. I. Lecture Notes in Mathematics. Springer-Verlag. Berlin. 339. (1973).

[LeTe] M. Lejeune-Jalabert, B. Teissier. Clôture intégrale des idéaux et equisingularité. Séminaire Lejeune-Teissier. Centre de Mathématiques École Polytechnique. (1974).

[Li] A. Liendo. Affine $\mathbb{T}$-varieties of complexity one and locally nilpotent derivations. Transform. Groups. 15. (2010). 389-425.

[LS] A. Liendo, H. Süss. Normal Singularities with Torus Actions. Tohoku Math. J. (to appear).

[LV] D. Luna, T. Vust. Plongements d'espaces homogènes. Comment. Math. Helv. 58. (1983). 186-245.

[NR] D.G. Northcott, D. Rees. Reductions of ideals in local rings. Proc. Cambridge. Philos. Soc. 50. (1954). 145-158.

[Od] T. Oda. Convex Bodies and Algebraic Geometry. An Introduction to the Theory of Toric Varieties. SpringerVerlag. Berlin. 131. (1985).

[RRV] L. Reid, L. G. Roberts, M. A. Vitulli. Some results on normal homogeneous ideals. Comm. in Alg. 31. (2003). 4485-4506. 
[Ri] P. Ribenboim. Anneaux de Rees intégralement clos. J. Reine Angew. Math. 204. (1960). 99-107.

[Ti] D. Timashev. Torus actions of complexity one. In : Toric topology. Contemp. Math. AMS. 60. (2008). 349-364.

[Ti 2] D. Timashev. Classification of G-manifolds of complexity 1. Izv. Ross. Akad. Nauk Ser. Mat. 61. (1997). $127-162$.

[Vie] E. Viehweg. Rational singularities of higher dimensional schemes. Proc. Amer. Soc. 63. (1977). 6-8.

[Vit] M. A. Vitulli. Some normal monomial ideals. Contemp. Math. 324. (2003). 205-217.

[Va] W. Vasconcelos. Integral closure: Rees algebras, multiplicities, algorithms. Springer. Monographs in Mathematics. XII. (2005). 519p.

[Vo] R. Vollmert. Toroidal embeddings and polyhedral divisors. Int. J. Algebra. 4. (2010). 383-388.

[ZS] O. Zariski, P. Samuel. Commutative Algebra. Van Nostrand. Princeton. Vol. II. (1960).

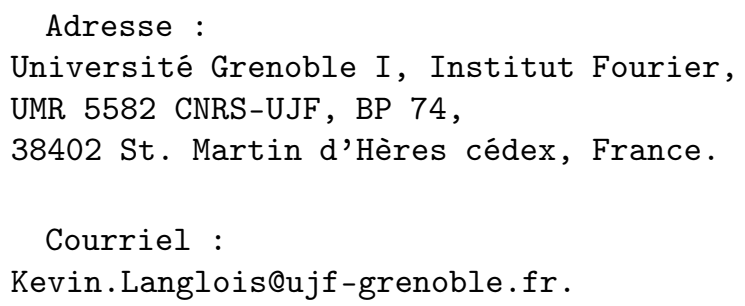

